\title{
Description of Resonant Inelastic X-Ray Scattering in Correlated Metals
}

\author{
Keith Gilmore $\odot,{ }^{1,}{ }^{*}$ Jonathan Pelliciari $\odot,{ }^{2,3,4, \dagger}$ Yaobo Huang, ${ }^{2,5}$ Joshua J. Kas, ${ }^{6}$ Marcus Dantz, ${ }^{2}$ Vladimir N. Strocov, ${ }^{2}$ \\ Shigeru Kasahara, ${ }^{7}$ Yuji Matsuda, ${ }^{7}$ Tanmoy Das, ${ }^{8}$ Takasada Shibauchi, ${ }^{9}$ and Thorsten Schmitt ${ }^{2, *}$ \\ ${ }^{1}$ Condensed Matter Physics and Materials Science Department, Brookhaven National Laboratory, \\ Upton, New York 11973, USA \\ ${ }^{2}$ Photon Science Division, Paul Scherrer Institut, CH-5232 Villigen PSI, Switzerland \\ ${ }^{3}$ Department of Physics, Massachusetts Institute of Technology, Cambridge, Massachusetts 02139, USA \\ ${ }^{4}$ National Synchrotron Light Source-II, Brookhaven National Laboratory, Upton, New York 11973, USA \\ ${ }^{5}$ Beijing National Lab for Condensed Matter Physics, Institute of Physics, \\ Chinese Academy of Sciences, Beijing 100190, China \\ ${ }^{6}$ Department of Physics, University of Washington, Seattle, Washington 98195, USA \\ ${ }^{7}$ Department of Physics, Kyoto University, Sakyo-ku, Kyoto 606-8502, Japan \\ ${ }^{8}$ Department of Physics, Indian Institute of Science, Bangalore 560012, India \\ ${ }^{9}$ Department of Advanced Materials Science, University of Tokyo, Kashiwa, Chiba 277-8561, Japan
}

(Received 3 November 2020; revised 19 March 2021; accepted 21 April 2021; published 19 July 2021)

To fully capitalize on the potential and versatility of resonant inelastic x-ray scattering (RIXS), it is essential to develop the capability to interpret different RIXS contributions through calculations, including the dependence on momentum transfer, from first principles for correlated materials. Toward that objective, we present new methodology for calculating the full RIXS response of a correlated metal in an unbiased fashion. Through comparison of measurements and calculations that tune the incident photon energy over a wide portion of the $\mathrm{Fe} L_{3}$ absorption resonance of the benchmark material $\mathrm{BaFe}_{2} \mathrm{As}_{2}$, we show that the RIXS response in $\mathrm{BaFe}_{2} \mathrm{As}_{2}$ is dominated by the direct-channel contribution, including the Raman-like response below threshold. Calculations are initially performed within the first-principles BetheSalpeter equation (BSE) framework, which we then significantly improve by invoking a quasiboson model to describe the secondary excitations within the intermediate state. This enhancement allows the many-electron RIXS signal to be approximated as a convolution of BSE-calculated spectra with effective spectral functions. We construct these spectral functions, also from first principles, by employing the cumulant expansion of the Green's function and performing a real-time time-dependent density functional theory calculation of the response of the electronic system to the perturbation of the intermediate-state excitation. Importantly, this process allows us to evaluate the indirect RIXS response from first principles, accounting for the full periodicity of the crystal structure and with full dependence on the momentum transfer.

DOI: 10.1103/PhysRevX.11.031013

\section{INTRODUCTION}

Resonant inelastic x-ray scattering (RIXS) is a highly versatile and powerful probe of elementary excitations in materials owing to its sensitivity to all electronic (i.e., spin, charge, and orbital) and lattice degrees of freedom,

\footnotetext{
*kgilmore@physik.hu-berlin.de pelliciari@bnl.gov

thorsten.schmitt@psi.ch
}

Published by the American Physical Society under the terms of the Creative Commons Attribution 4.0 International license. Further distribution of this work must maintain attribution to the author(s) and the published article's title, journal citation, and DOI.
Subject Areas: Condensed Matter Physics,

Strongly Correlated Materials elemental and orbital selectivity, detailed polarization analysis, and ability to detect small sample volumes [1,2]. RIXS is used to detect local and collective excitations such as $d d$ excitations [3,4], charge transfer [5-7], phonons [8,9], spin excitations [10-13], and other quasiparticles [14-16]. This sensitivity allows the study of the key interactions characterizing materials such as electronphonon coupling, magnetic exchange, fractionalization, and the interplay of emergent collective excitations. Recently, the technique has even been extended to timeresolved experiments [17,18]. The success of RIXS is due to the huge advancements in X-ray instrumentation [19-21] and the concomitant development of RIXS theory [22-26].

Experimental RIXS efforts focus to a large extent on strongly correlated electron materials, particularly Mott 
insulators. Correspondingly, computational efforts typically either utilize model Hamiltonians on 1D or 2D lattices [27-32] or treat small clusters around the absorbing element at various approximations [33-37]. The computational demands of both approaches scale prohibitively with system size, requiring truncation both with respect to the lattice or cluster size and with respect to the number of bands or active orbitals included in the calculation. These restrictions are tolerable for many of the transition metal oxides commonly probed thus far because of their insulating or narrow band character, nearly complete $d$ shell or subshell, and the typical reduced dimensionality. However, these computational limitations appear to preclude accurate treatment of more itinerant systems or even multiorbital nonmetallic materials with moderate bandwidth.

Multiorbital correlated materials present a rich physical landscape of interactions leading to a plethora of intriguing phenomena including spin, charge, and orbital orders, giant and colossal magnetoresistance, metal-to-insulator transitions, and multiferroicity [38,39]. Correlated metals offer several examples of unconventional superconductivity distinct from cuprates including intermetallic heavy fermion systems [40], the iron-based superconductors [41], and $\mathrm{Sr}_{2} \mathrm{RuO}_{4}$ [42]. Meanwhile, the narrow gap semiconductor $\mathrm{FeSb}_{2}$ [43] and ultrathin films of FeSe [44] possess exceptional thermoelectric properties. These multiorbital systems are subject not only to charge correlations, characterized by the Hubbard interaction, but also orbital correlations determined by Hund's coupling. The latter interaction leads to orbital differentiation and the possibility of orbital-selective Mott transitions [45]. The orbitalselective nature of correlations within Hund's metals and their interplay with Mott physics remains a fascinating and vigorously investigated subject $[46,47]$. While these properties are probed by other techniques such as angle-resolved photoemission [48,49], optical conductivity [50], and inelastic neutron scattering [45,51], applying RIXS to correlated metals offers several advantages such as bulk, elemental, and orbital sensitivity. However, the development of RIXS on these materials is hampered by the lack of a theoretical framework to interpret spectra of metallic and multiorbital systems, limiting the utility of RIXS.

Experimentally, the RIXS response of metals, and especially correlated metals, has been only marginally investigated due to the large fluorescence backgrounds that tend to obscure more interesting contributions from collective excitations such as phonons, magnons, and secondary charge excitations. Measurements of charge excitations in vanadates probe the influence of chemical composition [52] and film thickness [53] on electronic correlations. Magnon and paramagnon excitations are studied by RIXS in parent and doped $\mathrm{BaFe}_{2} \mathrm{As}_{2}$ compounds [54-57], in 111 [58] and 1111 [59] systems, and in FeSe [60]. However, the interpretation of RIXS data in multiorbital systems remains challenging.
Calculating the RIXS response of correlated metals is challenging, because it requires the ability to handle multiple orbitals, large bandwidths, and electronic correlations. One-electron or particle-hole methods, which are amenable to large bandwidths, are not well suited to accurately capture low-energy excitations of correlated systems and have largely been restricted to core-to-core RIXS processes [61,62]. Such calculations can account neither for secondary excitations in the direct RIXS channel nor for any part of the indirect RIXS contribution.

Here, we present a detailed, first-principles computational framework for evaluating and analyzing the valence electronic contributions to the RIXS spectra of metallic systems, taking the moderately correlated $\mathrm{BaFe}_{2} \mathrm{As}_{2}$ as our test case. Experimentally, we map the RIXS loss profiles of $\mathrm{BaFe}_{2} \mathrm{As}_{2}$ as a function of incident photon energy across the Fe $L_{3}$ edge. The most prominent feature is a Raman-tofluorescence crossover occurring around the x-ray absorption threshold. We provide a detailed analysis of the loss profiles, separating band-structure features from manybody contributions. For excitation energies well above the absorption edge, we observe a weak Raman-like feature at about $1 \mathrm{eV}$ energy loss that is not seen in previous studies. We interpret this feature as arising partly from electronic correlations within the $\mathrm{Fe} d$ bands.

Our computational work starts by solving the BetheSalpeter equation (BSE) for two-particle excitonic states based on an electronic band structure obtained from density functional theory (DFT). Contrary to model Hamiltonians, cluster calculations, or approaches that reduce the electronic structure to Wannier orbitals of the correlated bands, the DFT BSE includes all bands associated with all sites and orbital shells on an equal footing. This inclusion reduces the arbitrariness of the method, avoids parameterization, naturally accounts for hybridization effects, and is equally applicable to the transition metal and ligand edges. The BSE has previously been applied to calculate the x-ray absorption and emission spectra of metals [63], and the RIXS loss profiles of nonmetals [64-66], but generally not to the RIXS spectra of metals.

We show in Sec. II that the BSE, employing the typical static screening response, provides a reasonable accounting for spectral features observed in the experimental $\mathrm{x}$-ray absorption and emission spectra of $\mathrm{BaFe}_{2} \mathrm{As}_{2}$. However, certain discrepancies in the spectral intensity are evident. This discrepancy occurs because the BSE describes the excited state as a superposition of single electron-hole pairs and, due to the static screening approximation, fails to accurately capture spectral structures stemming from the generation of secondary excitations. We correct this deficiency by combining the BSE with a quasiboson model that accounts for shakeup within the valence system due to coupling to the core hole and photoelectron. This correction is accomplished in Sec. IV by using real-time timedependent density functional theory (rt-TDDFT) to calculate 
the dynamic, correlated response of the valence system due to the intermediate-state perturbation. This calculation of the correlated response allows us to generate, from first principles, an effective spectral function that implicitly incorporates the previously neglected secondary excitations. Convolving this spectral function with the BSE results yields spectra in excellent agreement with experiment.

The quasiboson model further admits a simplified expression of the RIXS cross section as a convolution over absorption and emission spectra. We show in Secs. V and VI that this approximation enables us to combine our BSE calculations and the rt-TDDFT-derived effective excitonic spectral function to calculate both band-structure and correlation contributions to RIXS spectra. This combination allows for a practical, first-principles method of calculating the RIXS response of correlated metals with full momentum dependence, including secondary electronic excitations and indirect loss contributions.

\section{X-RAY ABSORPTION AND EMISSION}

Before turning to the more complex RIXS results, we first consider the x-ray absorption (XAS) and $\mathrm{X}$-ray emission (XES) spectra to better understand the RIXS intermediate and final states and their relations to the unoccupied and occupied electronic structure of $\mathrm{BaFe}_{2} \mathrm{As}_{2}$. Figure 1(b) contains the measured and calculated $\mathrm{Fe} L_{3}$ XAS. The experimental spectrum displays a broad main peak with a maximum at an absorption energy around $707.7 \mathrm{eV}$ accompanied by a shoulder region between about 710 and $711 \mathrm{eV}$. This line shape is typical of metallic compounds and is similar to measurements performed on other Fe pnictides [54,55,58-60]. The good quality of the sample is evidenced by the lack of spurious distinct peaks associated with $\mathrm{Fe}^{3+}$ between 709 and $710 \mathrm{eV}$, which would be due to sample deterioration and oxidation.

We calculate the XAS from first principles by evaluating the two-particle excitonic Bethe-Salpeter equations within the OCEAN code [67]. The Bethe-Salpeter Hamiltonian (BSH) consists of independent particle terms for the photoelectron and core hole as well as an interaction kernel that includes the statically screened direct and bare exchange terms between the two particles [68]. The independent particle contributions are obtained from a density functional theory (DFT) calculation of the ground-state electronic band structure using the Quantum ESPRESSO code [69,70]. The BSE interaction kernel is incorporated by summing ladder diagrams to infinite order [71]. The BSE calculation captures each of the features observed in the experiment, though with some discrepancies in intensities, which we address below.

The BSE calculations reveal at least three features within the main peak, labeled $A, B$, and $C$, and several features in the shoulder region, collectively labeled $D$. To understand these contributions in more detail, we first consider in
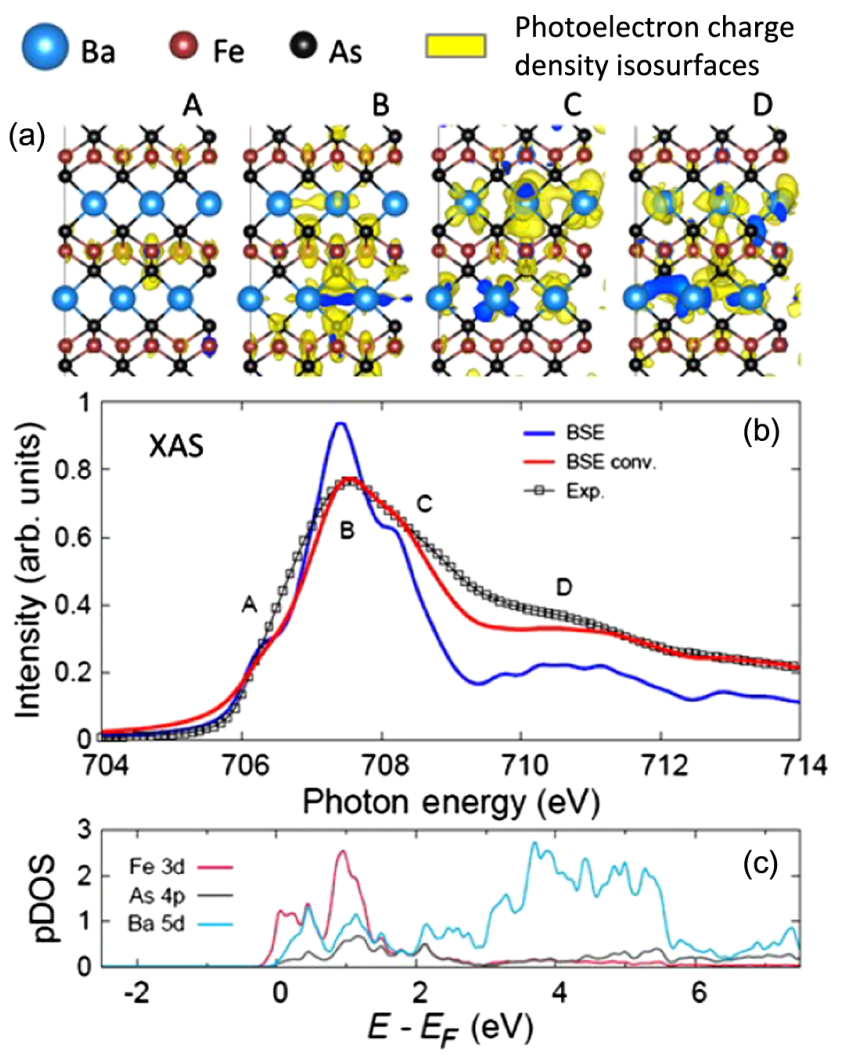

FIG. 1. X-ray absorption of $\mathrm{BaFe}_{2} \mathrm{As}_{2}$ at the $\mathrm{Fe} L_{3}$ edge. (a) Photoelectron charge density isosurfaces (yellow surfaces) for the BSE-calculated excitonic states $A-D$ indicated in the XAS spectrum; the core-hole component of the exciton wave function is fixed at a central iron atom. (b) BSE calculation (blue curve) of the XAS compared with measurement (black symbols). The corehole lifetime broadening is $0.2 \mathrm{eV}$ half width at half maximum (HWHM). The red curve is obtained as described in the text by convolving the BSE spectrum with a Doniach-Sunjic spectral function of asymmetry parameter 0.14 and linewidth $0.16 \mathrm{eV}$ that accounts for secondary electronic excitations. (c) Final-state local projected density of states (pDOS) of the unoccupied levels for selected orbitals.

Fig. 1(c) the final-state local projected density of states (pDOS) above the Fermi level around the absorbing Fe atom. The pDOS suggests that the edge onset $A$ is mainly composed of Fe $3 d$ and $\mathrm{Ba} 5 d$ states, the main peak $B$ is dominated by $\mathrm{Fe} 3 d$, hybridized to some extent with $\mathrm{Ba} 5 d$ and As $4 p$ states, the postedge $C$ contains appreciable Ba $5 d$ character, and the shoulder $D$ is mainly derived from $\mathrm{Ba} 5 d$ states. While the final-state pDOS, evaluated in the presence of a core hole on the absorbing Fe site, provides a valuable approximation of the orbital character of the final states, it does not account for weighting due to the transition matrix elements between the Fe $2 p$ core level and the final states nor for mixing of the final-state excitons caused by multipolar terms of the Coulomb interaction.

To provide a more rigorous picture, we visually inspect the excitonic states associated with the main edge and 
shoulder regions. For each incident photon energy $(A-D)$, we obtain the two-particle, electron-hole wave function for the excitonic XAS final state. Integrating out the core-hole coordinates leaves the wave function for the photoelectron. Figure 1(a) presents isosurfaces for the charge density distributions corresponding to these photoelectron wave functions. These orbital isosurfaces mainly confirm the pDOS interpretation, except that they indicate the pre-edge feature $A$ has very little Ba character, contrary to the pDOS prediction, and the shoulder region maintains more $\mathrm{Fe}$ character than implied in the pDOS. Consistent with the metallic nature of $\mathrm{BaFe}_{2} \mathrm{As}_{2}$, the photoelectrons are not strongly localized around the absorption site but are rather diffuse and delocalized. At the edge onset $(A)$, the photoelectron is largely confined within the first iron-arsenide layer, consisting of hybridized $\mathrm{Fe} 3 d$ and As $4 p$ states. By the postedge and shoulder regions, the photoelectron has a greater spatial expanse and gains appreciable $5 d$ orbital character on the Ba sites.

Figure 2(b) presents the measured and calculated XES at the $\mathrm{Fe} L_{3}$ edge. The experimental spectrum consists of a broad, asymmetric emission line; however, the calculation reveals finer structure with a main feature $A$ at around $706 \mathrm{eV}$, a distinct second feature $B$ near $703.5 \mathrm{eV}$, and a weak peak $C$ at $702 \mathrm{eV}$. Figure 2(c) gives the ground-state pDOS of the occupied states just below the Fermi level, showing that the main feature $A$ consists entirely of Fe $3 d$ orbitals, the shoulder feature $B$ is comprised of Fe $3 d-\mathrm{As}$ $4 p$ hybridized states, and the weak feature $C$ at $702 \mathrm{eV}$ is mainly of As $4 p$ character. This characterization is further supported by the orbital plots of the part of the excitonic wave functions corresponding to the final-state valence hole, which are shown in Fig. 2(a). To corroborate these assignments, we note that peaks $B$ and $C$, which derive appreciably from As orbitals, are absent in emission measurements of FeTe [72].

The BSE spectra are in reasonable agreement with the experimental results, though they are somewhat overstructured. The calculated XAS is too sharp at the main peak and lacks intensity in the postedge and shoulder regions. The comparison of the calculated to measured XES is similar. $\mathrm{BaFe}_{2} \mathrm{As}_{2}$ is considered a moderately correlated electron system [73], raising doubts [74] about the appropriateness of basing spectral calculations on a DFT electronic structure obtained with the local density approximation (LDA) to the exchange-correlation functional. However, the energies of all features correspond well between the calculated and measured XAS, suggesting that the underlying LDA band structure serves as a reasonable starting point for the BSE calculations. To further alleviate this concern, in Fig. 3 we compare the Fe $3 d$ pDOS obtained from LDA DFT and dynamical mean field theory (DMFT) calculations. The LDA DMFT calculation tends to sharpen the spectral function about the Fermi level but generally shifts spectral peaks by less than $1 \mathrm{eV}$. These changes on their own do not

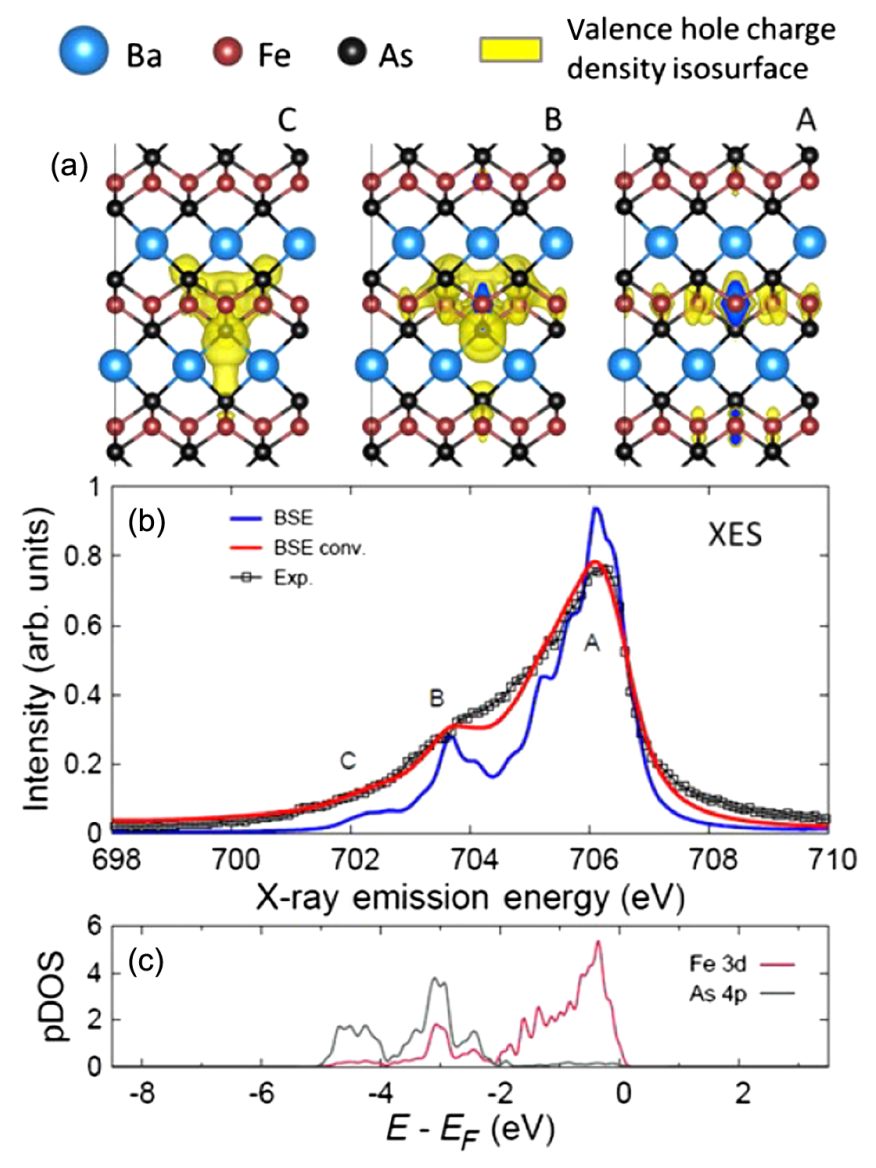

FIG. 2. X-ray emission of $\mathrm{BaFe}_{2} \mathrm{As}_{2}$ at the $\mathrm{Fe} L_{3}$ edge. (a) Orbital isosurface plots (yellow surfaces) of the valence-hole contribution for the BSE-calculated final state of the emission process corresponding to emission energies $A-C$ indicated in the main figure. (b) BSE-calculated XES (blue curve) with $0.2 \mathrm{eV}$ HWHM broadening compared with measurement (black symbols). The red curve is obtained as described in the text by convolving the BSE spectrum with a Doniach-Sunjic spectral function of asymmetry parameter 0.07 and linewidth $0.16 \mathrm{eV}$ that accounts for secondary electronic excitations. (c) Ground-state projected density of states for the occupied states of selected orbitals.

appear to explain the reduced intensity with respect to the experiment in the shoulder region of the XAS, which is primarily associated with the $\mathrm{Ba} 5 d$ orbitals.

The deviations in intensities of the spectral features could still be due to an inaccurate description of the spatial extent of the LDA Kohn-Sham wave functions; however, it is likely that a larger effect originates in the fundamental two-particle description of the BSE that neglects secondary electronic excitations beyond what is captured by the static screening response. The Hamiltonian $H_{\alpha c, \alpha^{\prime} c^{\prime}}^{\mathrm{BSE}}$ is expressed in a two-particle, electron-hole basis. For corelevel absorption, $\alpha$ denotes both the $m_{l}$ and $m_{s}$ values for the core hole, while $c$ incorporates the band index $n$, wave vector $\mathbf{k}$, and spin $\sigma$ of the conduction-level photoelectron. The excitonic eigenstates $\Omega^{\lambda}$ are obtained by 


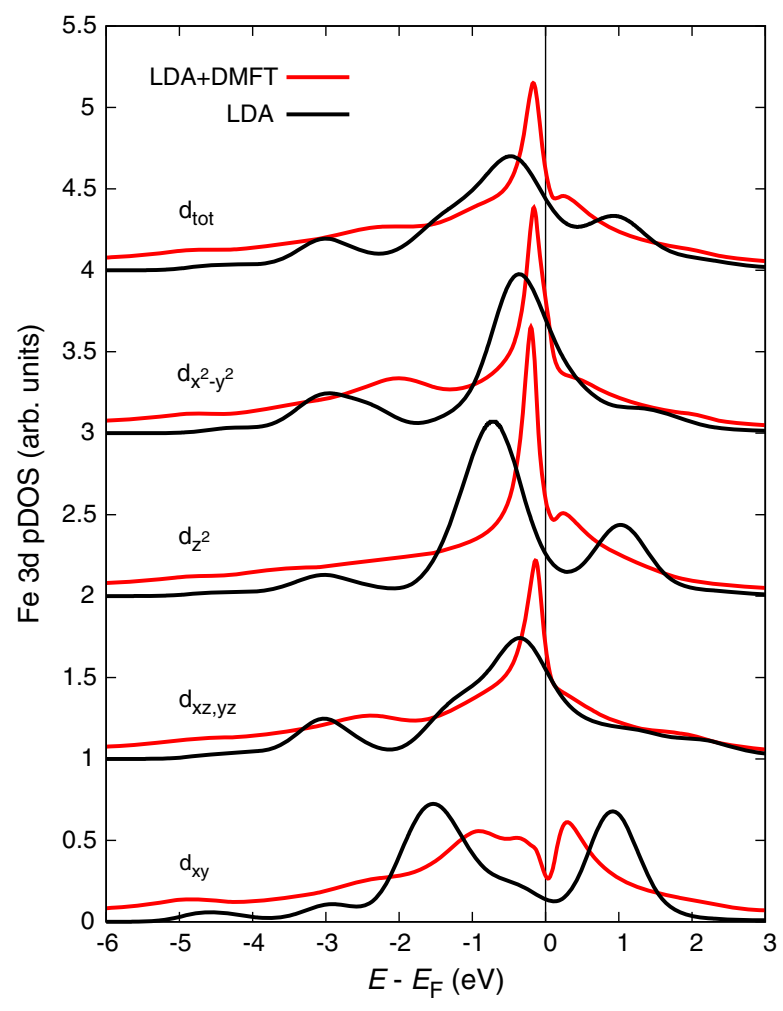

FIG. 3. Fe $3 d$ pDOS for $\mathrm{BaFe}_{2} \mathrm{As}_{2}$ calculated with LDA density functional theory (black) and dynamical mean field theory based on LDA DFT (red). Curves are offset vertically for clarity.

solving $\sum_{\alpha^{\prime} c^{\prime}} H_{\alpha c, \alpha^{\prime} c^{\prime}}^{\mathrm{BSE}} \Omega_{\alpha^{\prime} c^{\prime}}^{\lambda}=E^{\lambda} \Omega_{\alpha c}^{\lambda}$. These eigenstates can be expressed as linear superpositions of electron-hole pairs such that $\Omega^{\lambda}=\sum_{\alpha c} C_{\alpha c}^{\lambda}|\alpha, c\rangle$. The true many-body excited state will be more complex and involve the generation of additional, valence-conduction electron-hole pairs as the charge density responds to the creation of the core hole. We refer to these as the secondary excitations neglected within the BSE. Physically, these secondary excitations correspond to multiplet features in open-shell systems, as well as shakeup or shakeoff satellites and even plasmon satellites.

To some extent, it is possible to go beyond the single electron-hole pair description of the Bethe-Salpeter equation by explicitly constructing excited-state wave functions with a few secondary electron-hole pairs [75,76]. Instead, we prefer an implicit approach that effectively accounts for secondary excitations by expressing the many-body absorption coefficient

$$
\mu(\omega)=\sum_{\lambda} \int d \omega^{\prime} A_{\lambda}\left(\omega^{\prime}\right) \mu_{\lambda}^{0}\left(\omega-\omega^{\prime}\right)
$$

as a convolution of the single-exciton BSE absorption spectrum $\mu_{\lambda}^{0}$ with a spectral function $A_{\lambda}$ that accounts for these secondary excitations [77-79]. The spectral function $A_{\lambda}$ is specific to a particular excitonic eigenstate $\Omega^{\lambda}$ of the
BSH. X-ray photoemission studies of $\mathrm{BaFe}_{2} \mathrm{As}_{2}$ observe that the structure of the Fe $2 p$ core-hole spectral function is asymmetric and can be reproduced very well with a Doniach-Sunjic (DS) line shape [80], motivating the choice of a DS profile to approximate the effective spectral function $A_{\lambda}(\omega)$ in Eq. (1). In Sec. IV, we validate this assumption by explicitly constructing the spectral function from first principles. The expression for the DS line shape and the meaning of the parameters are provided in the Appendix D.

The XAS profile resulting from Eq. (1) [see the red curve in Fig. 1(b)] agrees much better with the experimental result than the bare BSE spectrum. Not only does the main peak trace the experimental profile very well, spectral weight is shifted from the main peak to the postedge region, leading to a significant improvement in the postedge and shoulder features. A small amount of spectral weight is still missing in the immediate postedge area around $709 \mathrm{eV}$. These small differences do not arise from experimental broadening of the XAS spectrum, as the energy resolution of the measurement is less than the corehole lifetime. The differences could potentially indicate minor shortcomings of the underlying LDA calculation. Alternatively, it is possible that the profile of secondary excitations is more complex than suggested by the Doniach-Sunjic line shape. In this case, local correlation effects on the Fe site could produce a more prominent $d d$ excitation in the range of $1-2 \mathrm{eV}$. We consider this possibility in more detail in Sec. VI.

Similarly to the XAS results, the BSE calculation for the emission spectrum is sharper than the measured spectrum with missing spectral weight below the main edge. Convolution of the BSE spectrum with a DS line shape again leads to considerably improved agreement with the experimental result, indicating that the emission process, i.e., filling the core hole, also incoherently kicks up various low-energy electron-hole pairs. However, the DoniachSunjic asymmetry parameter for emission $(0.07)$ is smaller than for absorption (0.14), suggesting that the effect, while still significant, is less pronounced in the emission case. This difference is potentially explicable in that, for XES, both initial and final states have a hole and no photoelectron, reducing the change in the potential between initial and final states compared to the XAS process.

\section{DIRECT-CHANNEL RESONANT INELASTIC X-RAY SCATTERING}

The Kramers-Heisenberg (KH) equation for the RIXS cross section is often expressed using explicit many-body intermediate $(M)$ and final $(F)$ states as

$$
\frac{d^{2} \sigma}{d \omega_{i} d \omega_{o}}=\frac{\omega_{o}}{\omega_{i}} \sum_{F}\left|\sum_{M} \frac{\left\langle F\left|\hat{\Delta}_{o}^{\dagger}\right| M\right\rangle\left\langle M\left|\hat{\Delta}_{i}\right| 0\right\rangle}{\omega_{i}-E_{M}+i \Gamma / 2}\right|^{2} \delta\left(\omega_{l}-E_{F}\right) .
$$


Setting the ground-state energy to zero, $E_{M}\left(E_{F}\right)$ is the energy of the intermediate (final) state, and $(\Gamma / 2)^{-1}$ is the intermediate-state lifetime. The transition operator $\hat{\Delta}_{i}\left(\hat{\Delta}_{o}\right)$ is associated with the incident (outgoing) photon, which has polarization vector $\hat{\epsilon}_{i}\left(\hat{\epsilon}_{o}\right)$, wave vector $\mathbf{k}_{i}\left(\mathbf{k}_{o}\right)$, and energy $\omega_{i}\left(\omega_{o}\right)$. The energy and momentum transferred during the scattering process are $\omega_{l}=\omega_{i}-\omega_{o}$ and $\mathbf{q}=\mathbf{k}_{i}-\mathbf{k}_{o}$, respectively. The $\mathrm{KH}$ formula in Green's function notation is

$\frac{d^{2} \sigma}{d \omega_{i} d \omega_{o}}=-\frac{1}{\pi} \frac{\omega_{o}}{\omega_{i}} \operatorname{Im}\left\langle 0\left|\hat{\Delta}_{i}^{\dagger} G^{\dagger}\left(\omega_{i}\right) \hat{\Delta}_{o} G\left(\omega_{l}\right) \hat{\Delta}_{o}^{\dagger} G\left(\omega_{i}\right) \hat{\Delta}_{i}\right| 0\right\rangle$.

The first and last Green's functions propagate the intermediate state in the presence of the core hole, while the central Green's function propagates the final, valenceexcited state.

Within the Bethe-Salpeter framework, the evaluation of Eq. (3) for the RIXS loss profile is separated into three steps. The process begins by generating the XAS-like intermediate core-excited state

$$
\left|y\left(\omega_{i}, \hat{\epsilon}_{i}\right)\right\rangle=G\left(\omega_{i}\right) \hat{\Delta}_{i}|0\rangle .
$$

The BSE reduces the many-body problem to an effective two-particle, electron-hole basis consisting of $\mathrm{Fe} 2 p$ core levels $\alpha$ and the unoccupied conduction states $c$. Approximating the propagator as $G(\omega)=\left[\omega-H^{\mathrm{BSE}}\right]^{-1}$, within this two-particle basis the RIXS intermediate (coreexcited) state is

$$
\left|y\left(\omega_{i}, \hat{\epsilon}_{i}\right)\right\rangle=\sum_{\alpha c, \alpha^{\prime} c^{\prime}}\left|\alpha^{\prime} c^{\prime}\right\rangle\left\langle\alpha^{\prime} c^{\prime}\left|\frac{1}{\omega_{i}-H^{\mathrm{BSE}}}\right| \alpha c\right\rangle\left\langle c\left|\hat{d}_{i}\right| \alpha\right\rangle,
$$

where we use the dipole approximation $\hat{d}=\hat{\epsilon} \cdot \mathbf{r}$. Evaluation of Eq. (5) requires solving a core-level BSE problem.

The second step of the RIXS calculation constructs the $\mathrm{x}$-ray emission state

$$
\left|x\left(\omega_{i}, \hat{\epsilon}_{i}, \hat{\epsilon}_{o}\right)\right\rangle=\hat{\Delta}_{o}^{\dagger}\left|y\left(\omega_{i}, \hat{\epsilon}_{i}\right)\right\rangle .
$$

This state is expressed within the two-particle description as

$$
\left|x\left(\omega_{i}, \hat{\epsilon}_{i}, \hat{\epsilon}_{o}\right)\right\rangle=\sum_{v} \sum_{\alpha c}|v c\rangle\left\langle v\left|\hat{d}_{o}^{\dagger}\right| \alpha\right\rangle\left\langle\alpha c \mid y\left(\omega_{i}, \hat{\epsilon}_{i}\right)\right\rangle,
$$

where $v$ runs over previously occupied valence states and represents a band index, wave vector, and spin direction. The vector $\left|x\left(\omega_{i}, \hat{\epsilon}_{i}, \hat{\epsilon}_{o}\right)\right\rangle$ contains a valence-conduction electron-hole pair, though this state is generally not an eigenstate of the RIXS final state. The final-state eigenstates and their corresponding RIXS intensities are obtained in the last step of the RIXS calculation by evaluating $\frac{d^{2} \sigma_{\mathbf{q}}}{d \omega_{i} d \omega_{o}} \propto-\operatorname{Im}\left\langle x\left(\omega_{i}, \hat{\epsilon}_{i}, \hat{\epsilon}_{o}\right)\left|\frac{1}{\omega_{l}-H_{\mathbf{q}}^{\mathrm{BSE}}}\right| x\left(\omega_{i}, \hat{\epsilon}_{i}, \hat{\epsilon}_{o}\right)\right\rangle$,

where this quantity now involves a valence-level BSE problem evaluated with finite momentum transfer q. We obtain the RIXS spectra using the OCEAN code $[64,67,81]$.

Figure 4(a) compares the RIXS profiles calculated by Eq. (8) to measurements for several incident photon energies around the $\mathrm{Fe} L_{3}$ edge of $\mathrm{BaFe}_{2} \mathrm{As}_{2}$. The most evident trend is the Raman-to-fluorescence crossover as the incident photon energy traverses the XAS peak. For incident energies tuned below the XAS threshold, the loss profile peaks around $1 \mathrm{eV}$ and extends out several $\mathrm{eV}$ to higher energy loss. This feature behaves in a Raman-like way; i.e., its position in energy loss is independent of the incident photon energy. However, as the incident photon energy passes the XAS threshold, the RIXS loss profile begins to behave in a fluorescencelike fashion; i.e., the features shift outward in energy loss proportionally with the increase of the incident photon energy. The calculations reproduce the experimental trends well, capturing both the specific loss profiles and the overall Raman-to-fluorescence crossover as the incident photon energy is scanned across the absorption threshold.

LDA tends to overestimate the bandwidth of correlated orbitals as seen in the comparison with the DMFT pDOS in Fig. 3. This overestimation causes excessive separation between the peaks of the occupied and unoccupied Fe $3 d$ pDOS, which exaggerates the energy loss of the calculated RIXS profiles. To correct for this error, the energy loss scale for the calculated spectra is shifted by $0.6 \mathrm{eV}$ to smaller absolute loss. This shift is attenuated as the energy loss approaches zero.

Fluorescence behavior is often associated with direct RIXS processes and Raman behavior with indirect processes. In this view, it is surprising that the calculations, which include only direct RIXS processes, capture both fluorescence and Raman behavior. This kind of Ramanto-fluorescence crossover is observed and discussed in resonant photoemission and resonant Auger spectroscopy [82-86] and has more recently been seen in RIXS [72,87]. The finite lifetime of the core hole allows below-threshold excitation, and, since the intermediate state is virtual, the energy loss depends only on the energy difference between the initial and final states. However, the excitation probability diminishes rapidly with detuning as observed in both the measurements and calculations. In Appendix C, we show that this attenuation becomes more pronounced as the core-level lifetime broadening decreases. Measuring the attenuation of the peak height in the Raman regime with respect to detuning below resonance could provide an experimental determination of the core-hole lifetime, though this quantification will likely be complicated by additional effects at resonance and by the presence of multiple resonance states close in energy. 


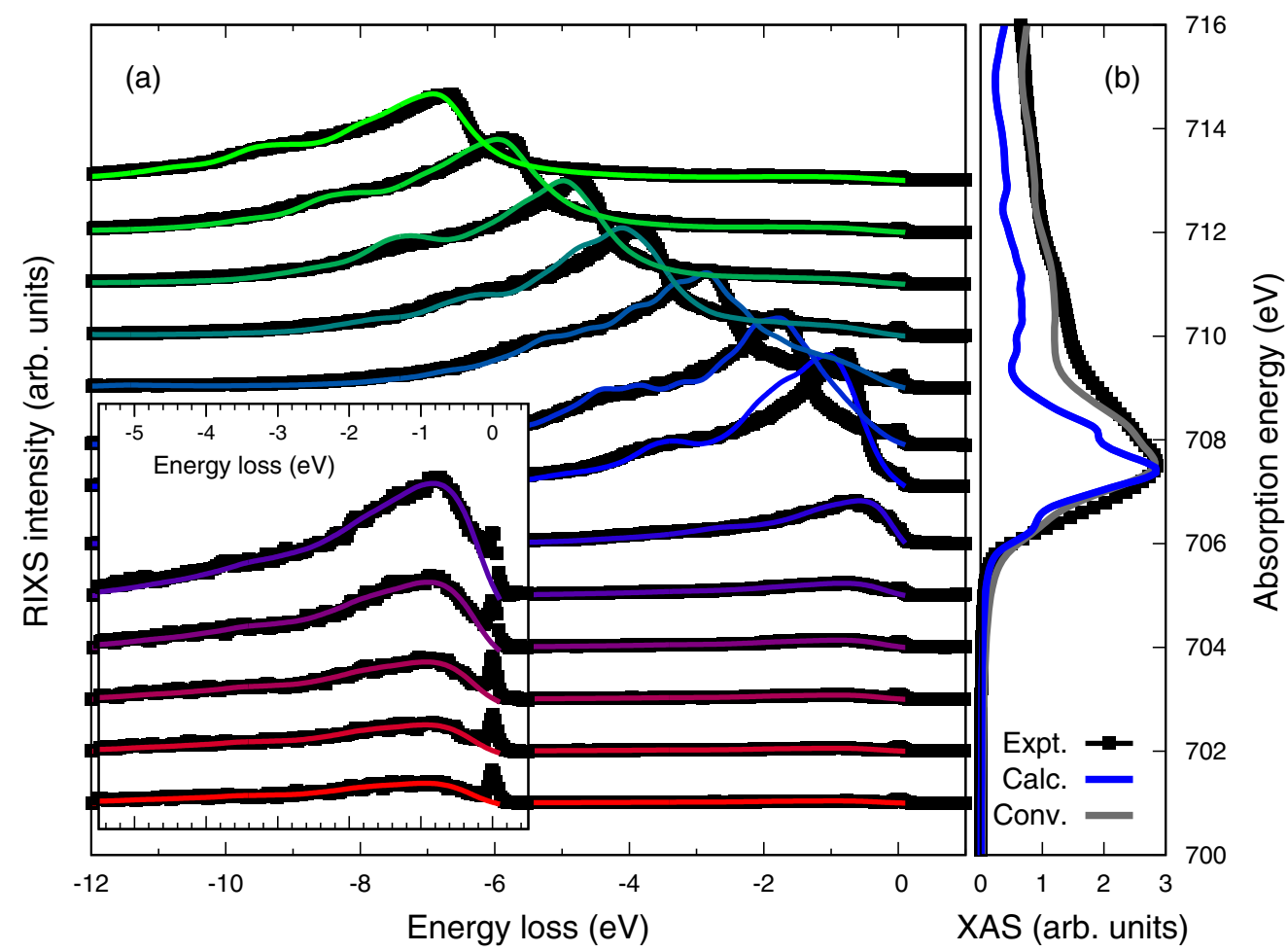

FIG. 4. (a) RIXS spectra at the Fe $L_{3}$ edge of $\mathrm{BaFe}_{2} \mathrm{As}_{2}$ plotted versus energy loss comparing BSE evaluation of the KramersHeisenberg equation (colored curves) to measurement (black symbols). Curves are staggered vertically to align with their corresponding incident photon energies with respect to the Fe $L_{3}$ XAS profile shown in (b). The inset in (a) expands the first $5 \mathrm{eV}$ of energy loss for the RIXS profiles with lowest incident photon energies. The momentum transfer for all spectra, experimental and calculated, is $(0.31$, $0.31,0)$ in reciprocal lattice units. Calculated loss profiles correspond to the evaluation of Eq. (8) using an intermediate-state core-hole lifetime equivalent to $0.2 \mathrm{eV}$ HWHM and a final-state broadening of $0.36 \mathrm{eV}$ HWHM. (b) Measured (black symbols) and BSEcalculated (blue curve) XAS at the Fe $L_{3}$ edge. The gray curve shows the BSE-calculated spectrum after convolution with a DoniachSunjic line shape and corresponds to the red curve in Fig. 1(b).

The main features of individual loss profiles appear consistent between the different incident energies and bear a strong resemblance to the $\mathrm{x}$-ray emission spectrum from Fig. 2. For all incident photon energies, there is a main loss peak followed after another $2-3 \mathrm{eV}$ of loss by a shoulder feature. A much weaker third feature can be seen about $1-2 \mathrm{eV}$ further in energy loss. There are minor differences in relative peak intensities as the incident photon energy is varied, but all loss profiles clearly reflect the XES. This result indicates that the RIXS process in $\mathrm{BaFe}_{2} \mathrm{As}_{2}$ is dominated by direct emission from occupied valence levels as opposed to the generation of secondary $d d$ or chargetransfer excitations. This description holds not just for the fluorescencelike region but also in the Raman-like region.

To obtain a more detailed view of the direct RIXS scattering process, Fig. 5 presents the electron and hole charge density contributions to the RIXS final-state valence-conduction excitons for several combinations of incident and outgoing photon energies. For incident photons tuned from below threshold to the peak of the absorption spectrum, the final-state electron charge density is largely confined within the FeAs layer. As the incident photon energy increases above the peak and into the tail of the $L_{3}$ edge, the electron charge density shifts more to the $\mathrm{Ba}$ layers. This shift mirrors closely the behavior of the excitonic states of the x-ray absorption spectrum in Fig. 1. Meanwhile, the orbital plots of the hole state in the valence band are approximately independent of the incident photon energy for each of the three main features in the RIXS loss profiles. This insensitivity indicates that, consistent with the metallic nature of the sample, excitonic effects are weak in the valence-excited RIXS final state. Consequently, the RIXS loss spectra are dominated by incoherent emission processes for all incident energies. These observations provide partial justification for simplifying the KramersHeisenberg expression in Sec. V.

Below the XAS threshold, Fig. 5 indicates that the excited electron in the RIXS final state occupies the same orbitals within the FeAs plane, just above the Fermi level. At the same time, the valence-hole orbitals are also independent of the incident photon energy for a given RIXS feature. Thus, for the Raman-like portion of the RIXS map, the final states consist of the same electron-hole pairs, generated through a direct scattering process. 


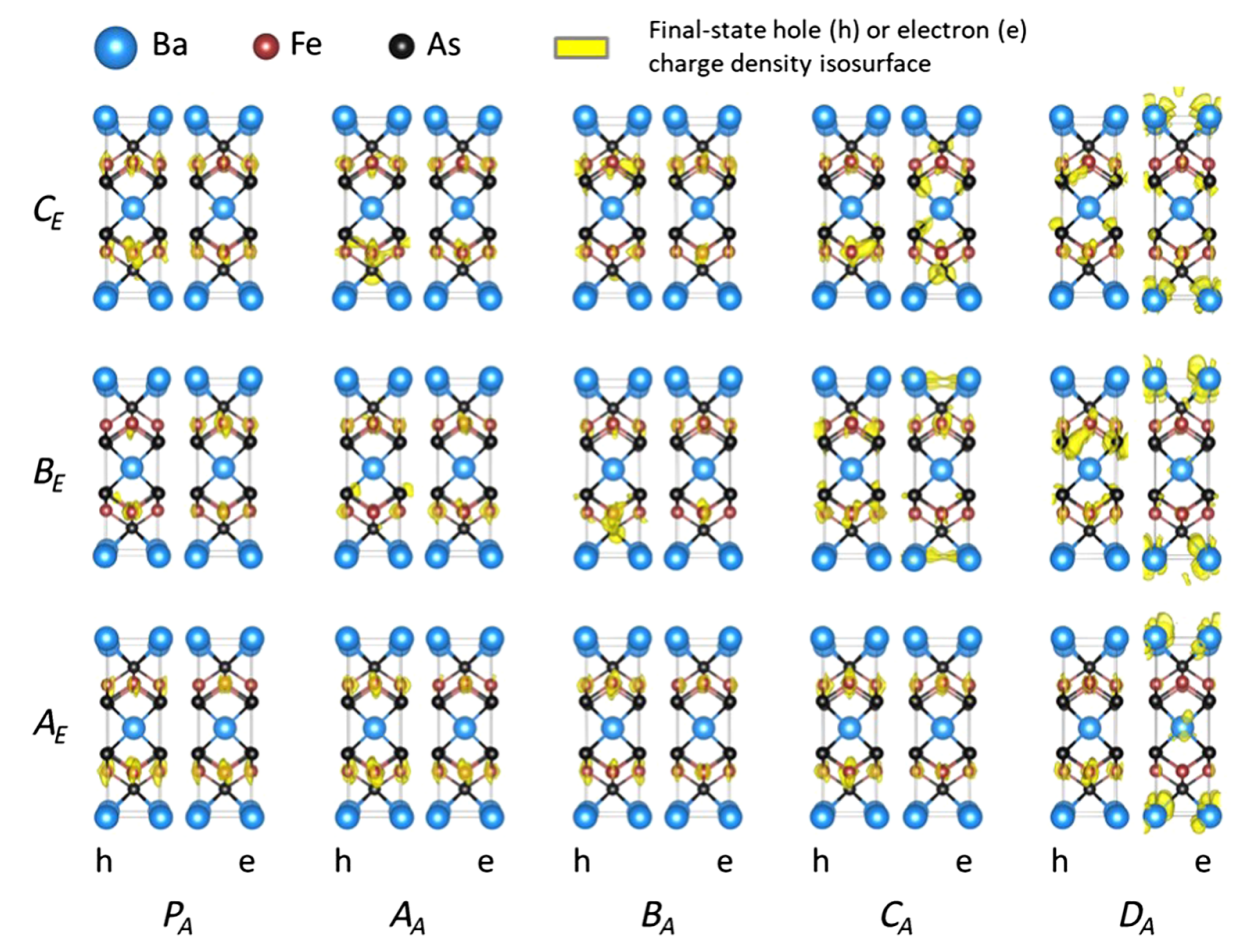

FIG. 5. BSE-calculated isosurface plots for the charge density of the hole $(h)$ and electron $(e)$ components of the RIXS final state exciton wave function for different combinations of absorption $\left(P_{A}, A_{A}, B_{A}, C_{A}, D_{A}\right)$ and emission features $\left(A_{E}, B_{E}\right.$, $\left.C_{E}\right)$; see labels in Figs. 1(b) and 2(b), respectively ( $P_{A}$ does not appear in Fig. 1 but corresponds to a pre-edge incident photon energy of $\left.705 \mathrm{eV}\right)$. For each combination of absorption and emission features, the left (right) isosurface plot corresponds to the hole (electron) component of the final-state exciton wave function.

\section{QUASIBOSON MODEL FOR SECONDARY EXCITATIONS}

It is typical to classify the RIXS response in terms of direct and indirect contributions. However, there is not a clear consensus as to the definition of these terms. From an empirical perspective, one is inclined to classify the fluorescence contribution as direct RIXS and Raman features, having constant energy loss, as the indirect contribution. This classification is qualitatively consistent with the canonical figures presented by Ament et al. [1]. In that work, the authors construct a precise, formal definition of the direct and indirect terms by expressing the intermediate-state propagator as the Dyson equation $G=G_{0}+G_{0} H_{C} G$, where $G_{0}$ is the ground-state propagator and $H_{C}$ introduces the core-hole (core-exciton) perturbation. The direct term is associated with the ground-state propagator $G_{0}$ and the indirect term with $G_{0} H_{C} G$. Within this definition, the direct contribution should be calculated neglecting all excitonic effects, meaning our BSE calculations would account for both direct and indirect responses, since we perform calculations using the full propagator, albeit an approximation thereof.

The above definitions are presented largely with Mott insulators in mind. At least when considering metals or systems for which the quasiparticle picture is not entirely lost, we prefer to introduce an alternative definition of the direct and indirect RIXS channels. The objective is to pragmatically separate band-structure contributions, underlying the direct signal, from the correlated response of the system to a perturbation, captured by the indirect contribution. Beginning with an independent electron approximation for the ground state, we define the direct contribution as that associated with excitation of a core electron to a state above the Fermi level and decay of another electron from a state below the Fermi level, filling the core hole. The populations of all other levels remain unaffected, though excitonic effects such as screening of the core hole and mixing of electron-hole pairs differentiate our definition from that offered by Ament et al. [1]. The indirect contribution arises when dynamic screening of the core-level exciton affects the populations of the remaining states through the generation of secondary excitations, e.g., shakeup or shakeoff processes. Collective excitations, such as plasmons, magnons, and phonons, could also appear in the indirect channel. The pure indirect channel derives from the recombination of the original photoelectron with the core hole. However, direct and indirect effects are not cleanly separable, and direct-channel processes may be accompanied by indirect-channel losses. Figure 6 presents a pictorial description of our definitions of the RIXS processes and associated excitations. 


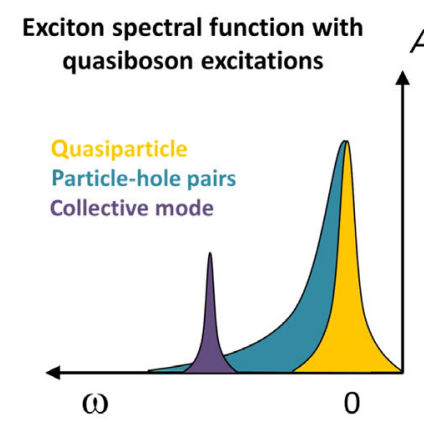

\section{$A(\omega)$}

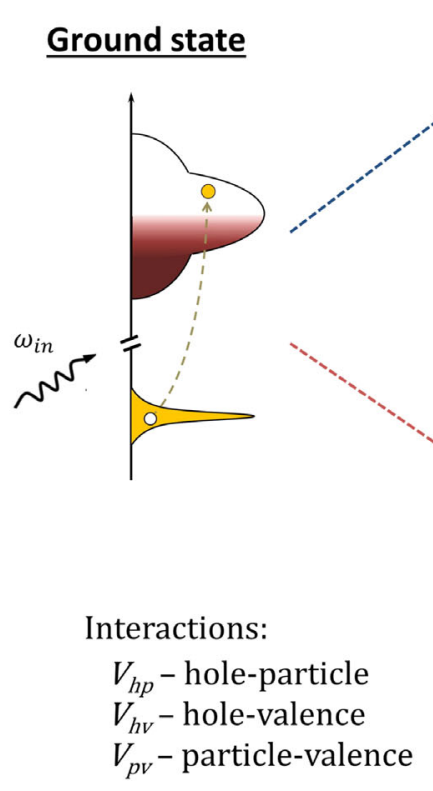

Intermediate states

Standard BSE
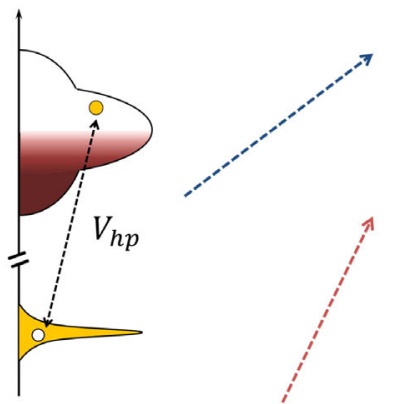

\section{Final states}
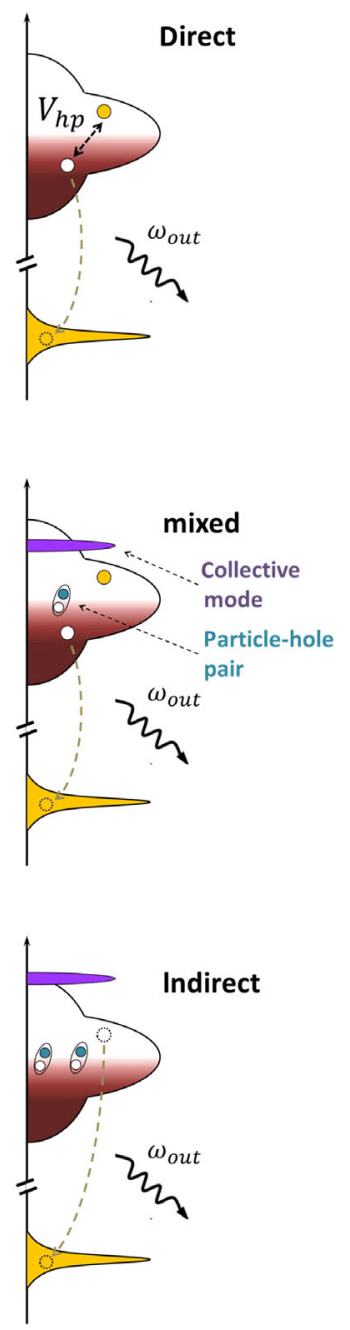

FIG. 6. Schematic diagram of the RIXS process within the standard BSE evaluation of the Kramers-Heisenberg formula (blue channel) and the BSE combined with a quasiboson Hamiltonian to describe secondary excitations of the valence levels (red channel). The standard BSE approach includes a screened interaction between the hole and photoelectron $\left(V_{h p}\right)$ in both the intermediate and final states but neglects explicit generation of additional excitations and, consequently, accounts for only pure direct RIXS processes. Combining the BSE with a quasiboson model that contains additional hole-valence $\left(V_{h v}\right)$ and photoelectron-valence $\left(V_{p v}\right)$ interactions in the intermediate state implicitly incorporates secondary excitations, allowing calculations to additionally generate indirect RIXS processes and mixed direct-indirect events. The top-left decomposes the effective exciton spectral function $A(\omega)$ into quasiparticle (yellow), particle-hole pairs (cyan), and collective modes (purple).

This modest redefinition associates the direct contribution with the primary core-hole-photoelectron exciton, treated at the quasiparticle level and neglecting any secondary excitations. This contribution is precisely what is evaluated by the BSE calculations taking the usual static screening approximation. This approximation fails to capture indirect RIXS contributions, since the intermediate and final states are explicitly restricted to a single hole plus single conduction electron basis. Indirect effects arise from all secondary valence-conduction excitations caused by the perturbation of the primary core exciton. Within the context of XAS and XES, in Sec. II, we demonstrate the efficacy of going beyond the single electron-hole pair description of the BSE by utilizing effective spectral functions to account for secondary excitations. In this section, we explicitly construct the XAS spectral function from first principles, and in Sec. V we develop an extension of our BSE calculations that uses the excitonic spectral function to implicitly capture the indirect RIXS channel.

The response of a metallic system to the sudden creation of a core hole was initially theoretically investigated at the end of the 1960s in what has become known as the MahanNozières-de Dominicis (MND) model [88-90]. The MND model accounts for many-body effects and the generation 
of secondary excitations, such as electron-hole pairs and plasmons, by synthesizing two competing effects: the Anderson orthogonality catastrophe [88] and Mahan's edge singularity [89]. The orthogonality catastrophe states that the many-body final state (in the presence of the core hole) is orthogonal to the initial (ground) state and, therefore, the transition intensity should go to zero at threshold. Meanwhile, the edge singularity predicts an infinite transition probability due to the generation of secondary electron-hole pairs with vanishing energy at the Fermi level in a metal. The MND theory combines the two ideas, yielding a finite absorption intensity at threshold.

While the MND theory is formally exact for the sudden creation of a deep core hole, to extend the theory to include the photoelectron and to obtain numerical results, Bardyszewski and Hedin introduced a quasiboson model [91-93]. The quasiboson model for core excitations separates the $N$-electron system into a core level $(h)$, a photoelectron $(p)$, and an $(N-1)$-electron valence system $(v)$. Interactions are included between the three subsystems giving the Hamiltonian

$$
H=H_{v}+h_{h}+h_{p}+V_{h v}+V_{p v}+V_{h p} .
$$

The hole, photoelectron, and their interaction $\left(V_{h p}\right)$ may be treated at the BSE level. Excitations of the valence electron system are described as quasibosons, such as electron-hole pairs or plasmons, so that $H_{v}=\sum_{q} \omega_{q} \hat{b}_{q}^{\dagger} \hat{b}_{q}$, where $\hat{b}_{q}^{\dagger}$ is a bosonic creation operator for a mode $q$. These quasibosonic modes are excited by interaction with the core hole $\left(V_{h v}\right)$ and the photoelectron $\left(V_{p v}\right)$ and are the secondary excitations responsible for the indirect losses in RIXS. One typically refers to the core-hole perturbation as the intrinsic effect and excitations caused by the photoelectron as extrinsic effects. Interference between these two interactions has been studied [77,93].

As we summarize in Appendix A, the many-body x-ray absorption coefficient can be reduced to a convolution of the BSE absorption coefficient and a spectral function that corresponds to the imaginary part of an effective twoparticle Green's function for the exciton. This two-particle Green's function implicitly accounts for the correlated response of the many-electron system. To generate a spectral function with suitable structure, we express the Green's function in the cumulant representation $G(t)=$ $G_{0}(t) e^{C(t)}$ and follow the work of Kas et al. to construct the cumulant $C(t)$ from first principles [77,94,95]. The bare two-particle Green's function for the exciton $G_{0}$ is obtained from the solution of the BSE as discussed in Sec. III. To second order, the cumulant is

$$
C(t)=\int d \omega \frac{\beta(\omega)}{\omega^{2}}\left(e^{-i \omega t}+i \omega t-1\right),
$$

where the quasiboson excitation spectrum of the valence electron system is given by

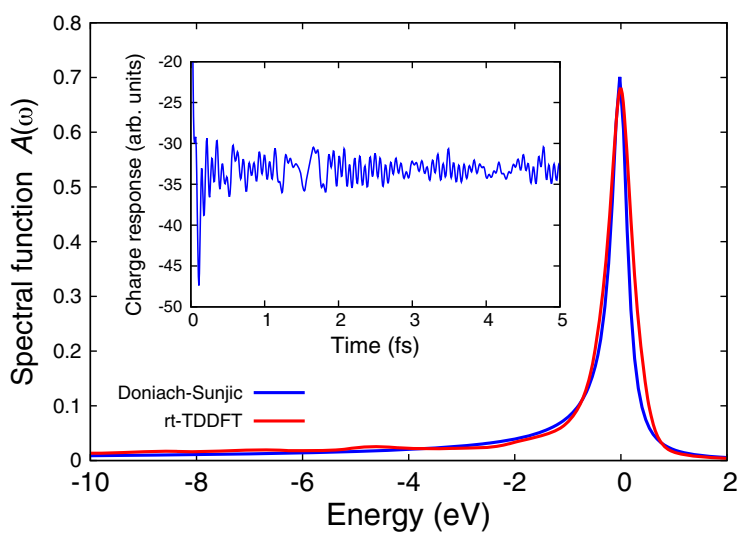

FIG. 7. Spectral function for the Fe $2 p$ core-excited state in $\mathrm{BaFe}_{2} \mathrm{As}_{2}$ due to secondary electronic excitations. The red curve is the result from a rt-TDDFT calculation as described in the text. The blue curve is a Doniach-Sunjic line shape with an asymmetry parameter of 0.14 and a linewidth of $0.16 \mathrm{eV}$. Inset: real-time valence charge density response to the sudden creation of a core hole at a $\mathrm{Fe}$ site.

$$
\beta(\omega)=\sum_{q, q^{\prime}} V_{q}^{*} V_{q^{\prime}} \operatorname{Im}\left[\chi\left(q, q^{\prime} ; \omega\right)\right]
$$

and the valence charge density response function is

$$
\chi\left(q, q^{\prime} ; \omega\right)=i \int_{0}^{\infty} d t e^{i \omega t}\left\langle\rho_{q}(t) \rho_{q^{\prime}}(0)\right\rangle .
$$

$V_{q}$ are the fluctuation potentials that generate the secondary excitations of the valence system of mode $q$ and are constructed as the Fourier components of the screened potential associated with the exciton.

To construct the charge density response function $\chi\left(q, q^{\prime} ; \omega\right)$, we perform a real-time time-dependent density functional theory calculation of the valence charge density response $\rho(t)$ to the potential created by the core-excited state. Optimally, one should build the perturbation $V_{q}$ from the full exciton wave functions of the combined core hole and photoelectron, as recently proposed [96]. In this case, separate rt-TDDFT calculations must be performed for each BSE eigenstate $\Omega^{\lambda}$, resulting in unique spectral functions $A_{\lambda}$ for each feature in the XAS spectrum. To simplify our present demonstration, we follow Woicik et al. $[78,79]$ and use the potential of only the core hole in the rt-TDDFT response calculation and obtain a single spectral function for all features in the XAS spectrum. For metallic systems, we expect this approximation to be fair; however, it could be improved upon in future work by using the full exciton potential for each BSE eigenstate.

The density response in the time domain and the resulting spectral function are shown in Fig. 7. The spectral function has very little structure beyond an asymmetric tail and can be approximated well with a Doniach-Sunjic line shape having an asymmetry parameter of 0.14 . This result 
indicates that the secondary excitations created during the $\mathrm{x}$-ray absorption process consist overwhelmingly of lowenergy electron-hole pairs rather than distinct, localized $d d$ excitations or higher-energy plasmons.

The ability to calculate effective spectral functions from first principles using the combination of the quasiboson model, rt-TDDFT, and the cumulant expansion significantly improves the agreement between calculated and measured spectra without introducing any ad hoc parameters. The technique was recently used to correctly identify excitonic, charge-transfer, and shakeup features in XAS and XPS spectra at both the $K$ and $L$ edges of transition metal compounds $[78,79]$. In the next two sections, we use the cumulant spectral function to consider the impact of secondary excitations on RIXS spectra. In particular, we provide a route to generating the indirect RIXS contributions from a band-structure perspective.

\section{INCORPORATING SECONDARY EXCITATIONS WITHIN RIXS CALCULATIONS}

Representing the full many-body absorption and emission spectra as convolutions of the BSE spectra and spectral functions as in Eq. (1) offers both qualitative and quantitative advantages. As shown in Sec. II, it allows for far more accurate XAS and XES spectra of periodic systems based entirely on first-principles calculations, the accuracy of which may be systematically improved. Use of manybody spectral functions also allows us to study the impact of secondary excitations on the direct RIXS contribution and opens the possibility of constructing an effective description of the indirect RIXS channel, which has previously been beyond the reach of first-principles band-structure-based calculations.

Compared to insulating systems, metals offer advantages for reasonably simplifying the full Kramers-Heisenberg equation. First, excitonic effects in the final state are weak, which allows us to treat the photoelectron as independent of the emission process. This approximation is supported by the orbital plots in Fig. 5 and the comparison of the BSE evaluation of the full $\mathrm{KH}$ formula [Eq. (8)] with and without final-state excitonic interactions shown in Appendix B (Fig. 12). Second, near threshold, the photoelectron in a metallic system experiences considerable scattering with the valence electrons, which further reduces the coherence between the absorption and emission processes. These considerations have previously been used to approximate the direct RIXS loss profile as a convolution of the XAS and XES spectra $[61,62]$ :

$$
\sigma_{D}\left(\omega_{i}, \omega_{o}\right)=\int d \tilde{\omega} \frac{\mu_{a}(\tilde{\omega}) \mu_{e}\left(\tilde{\omega}-\omega_{l}\right)}{\left(\omega_{i}-\tilde{\omega}\right)^{2}+(\gamma / 2)^{2}} .
$$

Equation (13) neglects any coherence between the absorption and emission processes except for evaluating the emission intensity at an energy lying $\omega_{l}$ below the intermediate-state exciton energy. A summary of the derivation of Eq. (13) is presented in Appendix B, and its suitability should hold across the iron pnictides and chalcogenides as well as more metallic systems.

Equation (13) also provides an approximation to the indirect RIXS response. As in Eq. (1), we expand the full spectrum $\mu_{a}\left(\mu_{e}\right)$ as a convolution over the bare BSE spectrum $\mu_{a}^{0}\left(\mu_{e}^{0}\right)$ and the spectral function $A_{a}\left(A_{e}\right)$. Secondary excitations can be created during both the absorption and emission events; however, in $\mathrm{BaFe}_{2} \mathrm{As}_{2}$, the $\mathrm{x}$-ray absorption process evidently generates secondary excitations to a greater extent than the emission process, so we consider only energy loss originating from secondary excitations occurring during the absorption step. Correspondingly, we replace the full XES spectrum $\mu_{e}$ with the bare BSE spectrum $\mu_{e}^{0}$. This replacement gives the indirect term as

$$
\sigma_{I}\left(\omega_{i}, \omega_{o}\right)=\int d \tilde{\omega} \frac{\left[\mu_{a}^{0}\left(\tilde{\omega}-\omega_{l}\right)\right]^{2} A_{a}\left(\omega_{l}\right)}{\left(\omega_{i}-\tilde{\omega}\right)^{2}+(\gamma / 2)^{2}} .
$$

Since, for the indirect contribution, the state involved in the emission process is the conduction band photoelectron state created during the absorption step, the emission factor $\mu_{e}^{0}$ has been replaced with a second absorption factor $\mu_{a}^{0}$ with the same energy argument.

An advantage of Eqs. (13) and (14) is that they simplify the incorporation of secondary excitations into firstprinciples calculations. Specifically, taking the absorption (emission) spectra $\mu_{a}\left(\mu_{e}\right)$ as the convolutions in Eq. (1), one can construct an effective, first-principles many-body RIXS response for a periodic system by calculating the one- or two-particle absorption (emission) spectra $\mu_{a}^{0}\left(\mu_{e}^{0}\right)$, e.g., with the BSE, and then generating corresponding spectral functions $A$ as described in the previous section. To simplify the presentation, we assume Doniach-Sunjic line shapes for both the absorption $\left(A_{a}\right)$ and emission $\left(A_{e}\right)$ spectral functions within this section. Based on the DS fit to the rt-TDDFT spectral function shown in Fig. 7, we use an asymmetry parameter of 0.14 and a linewidth of $0.16 \mathrm{eV}$ for the absorption spectral function. For the emission spectral function, we reduce the asymmetry parameter to 0.07 while maintaining the linewidth of $0.16 \mathrm{eV}$, based on the favorable convolution result found in Fig. 2.

Results for the direct and indirect RIXS contributions based on Eqs. (13) and (14) are presented in Figs. 8(a) and 8(b), respectively. The direct term reproduces well the trends seen in both experiment and calculations of the full Kramers-Heisenberg formula. Compared to the latter, the incorporation of secondary excitations generally shifts spectral weight to higher-energy losses, improving agreement with experiment similarly to the XES spectra shown in Fig. 2. Given the featureless structure of the DoniachSunjic spectral function, the indirect RIXS signal consists mainly of a Raman-like quasielastic line with a tail that 

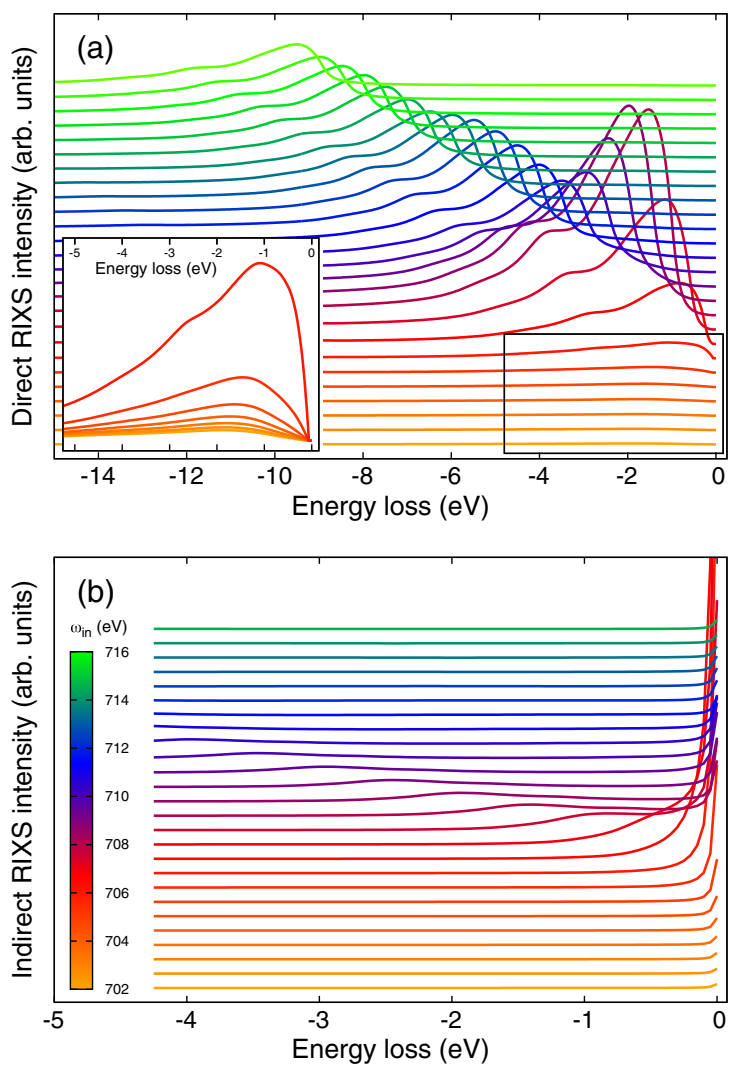

FIG. 8. Calculation of the direct (a) and indirect (b) RIXS contributions at the $\mathrm{Fe} L_{3}$ edge of $\mathrm{BaFe}_{2} \mathrm{As}_{2}$ according to Eqs. (13) and (14), respectively. Loss profiles for selected incident photon energies, from 702.5 to $715 \mathrm{eV}$ in steps of $0.5 \mathrm{eV}$, are displaced vertically. The color bar designating the incident photon energy in (b) applies in (a) as well. The calculations of the direct channel (a) reproduce the Raman-tofluorescence crossover at the absorption threshold. The inset provides an expanded view of the first $5 \mathrm{eV}$ of loss for the belowthreshold Raman-like region. Within the indirect channel (b), the elastic line extends to finite energy loss related to secondary electron-hole pair excitations captured by the quasiboson spectral function. Calculations use Doniach-Sunjic spectral functions as described in the text. A core-hole lifetime equivalent to $0.2 \mathrm{eV}$ HWHM broadening is applied to the intermediate-state resonance. The unexpected fluorescence feature in (b) is discussed in the text.

extends to finite energy loss. The intensity of the quasielastic feature varies with the absorption profile. A subtle fluorescencelike feature is also clearly present. This behavior originates in the nonuniform absorption intensity across the Fe $L$ edge. Because of the much greater absorption intensity at the main peak compared to the postedge region, secondary excitations associated with excitonic states at the main peak make sizable contributions to the loss profile. As the incident photon energy is tuned further above the main edge, the energy of the secondary excitations associated with the main edge increases accordingly, causing an apparent fluorescencelike behavior for the loss peak.

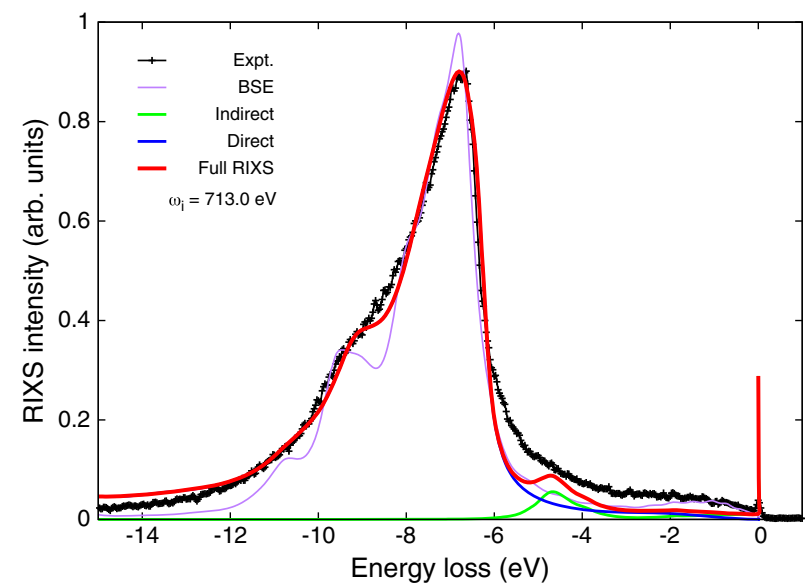

FIG. 9. Measured and calculated full RIXS profile for $\mathrm{BaFe}_{2} \mathrm{As}_{2}$ for an incident photon energy of $713.0 \mathrm{eV}$. Direct (blue curve) and indirect (green curve) contributions, calculated by Eqs. (13) and (14), respectively, are shown in addition to the full RIXS signal (red curve) with comparison to the BSE result for the full Kramers-Heisenberg equation (violet curve) and experimental result (black curve). Calculations use a core-hole lifetime of $0.2 \mathrm{eV} \mathrm{HWHM}$ and a Doniach-Sunjic spectral function with $0.16 \mathrm{eV}$ linewidth and 0.14 asymmetry parameter.

This effect has potentially been observed experimentally in superlattices of LAO and STO [97].

Figure 9 shows that the RIXS calculations including secondary excitations reproduce the experimental results very well, improving agreement compared to the regular BSE calculation of the Kramers-Heisenberg equation. This agreement further demonstrates that the large majority of the RIXS signal occurs through the direct channel and additionally validates the approximations made in Eqs. (13) and (14). Interestingly, the experimental result shows additional intensity compared to the direct contribution around 3-5 eV. Here, the fluorescencelike contribution to the indirect signal augments the direct contribution, noticeably improving the agreement with experiment. This fluorescence feature is overstructured in our calculation due to restricting all losses to the absorption step. Partitioning losses between absorption and emission processes would smooth out this contribution. As in Fig. 4, we correct for the LDA overestimation of the Fe $3 d$ bandwidth by shifting the energy loss scale for all calculations by $0.6 \mathrm{eV}$, as suggested by comparison to the DMFT pDOS.

\section{OBSERVATION OF $d d$ EXCITATIONS IN $\mathrm{BaFe}_{2} \mathrm{As}_{2}$}

A hallmark of strongly correlated electron systems is the loss of quasiparticle coherence and the emergence of Hubbard sidebands. In terms of photoemission, the sudden removal of an electron is typically accompanied by the generation of secondary excitations that appear as satellite features to the main spectral line with intensities determined by the quasiparticle renormalization factor. 


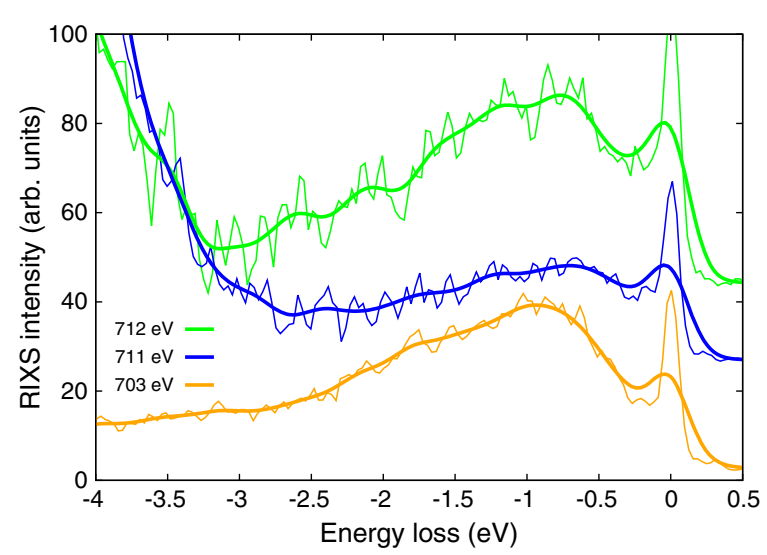

FIG. 10. Comparison of the excitonic peak in measured RIXS loss profiles of $\mathrm{BaFe}_{2} \mathrm{As}_{2}$ for incident photon energies below and above the absorption edge. The spectra are offset and smoothed (heavy lines) for better visualization. A linear fluorescence background is subtracted from the above-threshold curves.

However, $\mathrm{x}$-ray photoemission of $\mathrm{BaFe}_{2} \mathrm{As}_{2}$ does not show clear satellites [80], and only recently have orbital excitations in Fe-based superconductors been observed by RIXS [98]. The lack of obvious pure indirect Raman features in the RIXS profiles of Fe pnictides is seemingly at odds with their correlated nature. Such features would be expected to appear at low energy loss, within the localized iron $d$ bands. Close inspection of our experimental results reveals the persistence of a faint feature around $1-2 \mathrm{eV}$ at high incident energy, as shown in Fig. 10.

The nature of this feature is somewhat unclear. On the one hand, well above threshold, this weak peak resembles the direct fluorescence seen in the below-threshold Raman regime. This observation suggests it may also be a lifetimeallowed detuning remnant of the main resonance but for positive detuning. Indeed, our direct RIXS calculations using the Kramers-Heisenberg formula show weak intensity at this loss energy well above threshold (see Fig. 9, violet curve). On the other hand, this feature does not attenuate as expected with positive detuning. Rather, it appears even stronger at $712 \mathrm{eV}$ incident photon energy than at $711 \mathrm{eV}$. Thus, this feature is likely a combination of a lifetime-allowed direct-channel contribution from the main fluorescence peak and a true $d d$ indirect contribution.

Within our computational scheme, indirect effects are captured by the excitonic spectral function, which we generate through a rt-TDDFT calculation. This calculation yields a Doniach-Sunjic-type line shape that matches the experimental XPS of $\mathrm{BaFe}_{2} \mathrm{As}_{2}$ and leads to quite favorable comparison with the XAS after convolution with the BSE spectrum. However, this line shape does not produce a distinct $d d$ excitation in the indirect RIXS response around 1-2 eV, and the XAS shows some missing intensity about 1-2 eV above the main peak. One likely source for this discrepancy is that we considered only the core-hole potential when generating the spectral function. Properly including the contribution of the photoelectron as well may produce the missing $d d$ excitation. Also, it is conceivable that our TDDFT calculation, using the generalized gradient approximation (GGA) to the exchange-correlation functional, might fail to capture certain on-site correlated responses for $\mathrm{BaFe}_{2} \mathrm{As}_{2}$ that would contribute a clear $d d$ peak to the otherwise featureless DS profile. In either case, it is instructive to consider the RIXS spectra resulting from a spectral function that is more structured, having a distinct $d d$ excitonic satellite.

We present this case in Fig. 11 by combining the bare BSE absorption and emission spectra $\left(\mu_{a}^{0}\right.$ and $\left.\mu_{e}^{0}\right)$, calculated specifically for $\mathrm{BaFe}_{2} \mathrm{As}_{2}$, with an artificially adjusted
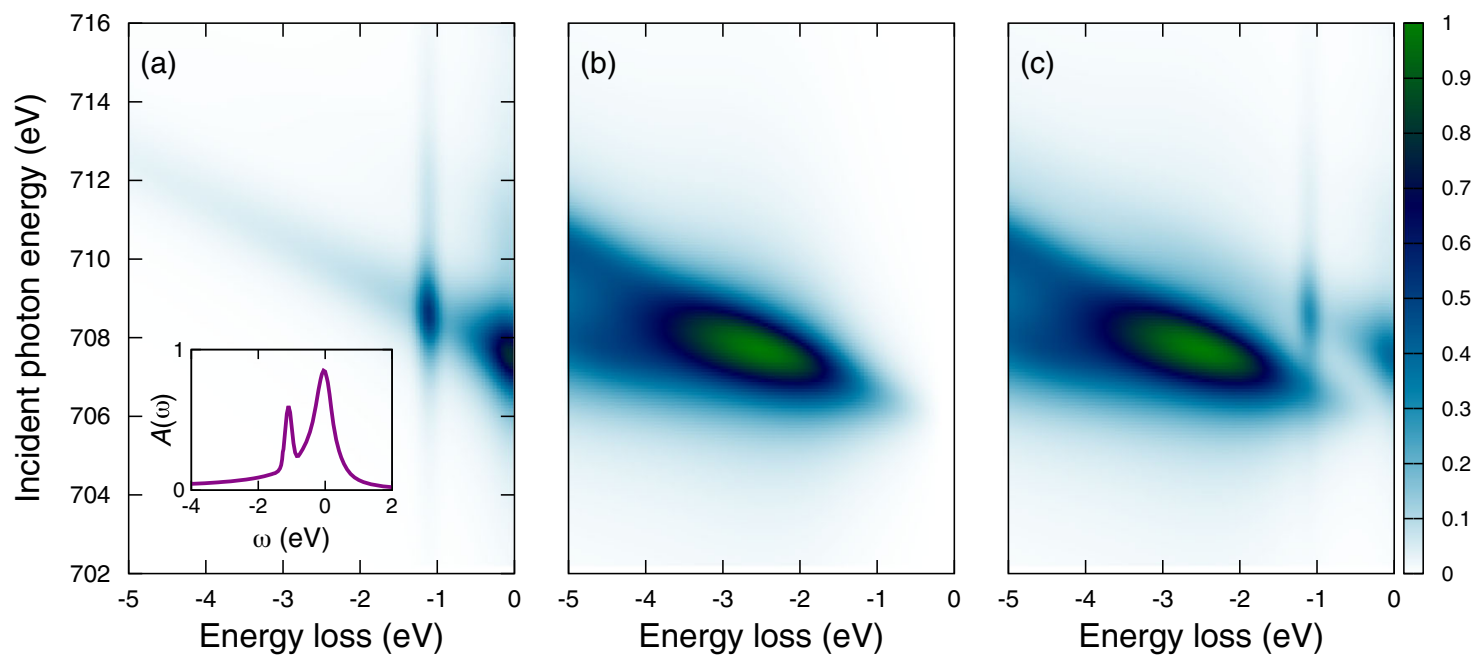

FIG. 11. Calculated RIXS maps for $\mathrm{BaFe}_{2} \mathrm{As}_{2}$ using the model spectral function shown in the inset in (a) in conjunction with the approximations made in Eqs. (13) and (14). The panels show the indirect contribution (a), direct contribution (b), and the full RIXS signal (c). The intensity of the indirect contribution in (a) is multiplied by 2 for visual clarity. The model spectral function consists of a Doniach-Sunjic profile with the addition of a satellite peak at $1.1 \mathrm{eV}$ representing a local $d d$ excitation. 
spectral function comprised of the previous DS profile with an additional satellite peak split off by $1.1 \mathrm{eV}$ [see the inset in Fig. 11(a)]. The indirect contribution [Fig. 11(a)] consists of a true elastic feature at zero energy loss and its fluorescent extension, as in Fig. 8(b), and a weaker Raman feature at $1.1 \mathrm{eV}$ loss associated with the $d d$ satellite added to the spectral function. We note that the maximum in intensity of the $1.1 \mathrm{eV}$ Raman loss feature occurs for an incident energy $1.1 \mathrm{eV}$ above the peak of the elastic line (i.e., it is fluorescence shifted). This model result resembles the measured spectra in the low loss region well above threshold, supporting the argument that the loss feature observed at approximately $1-2 \mathrm{eV}$ contains a contribution from a $d d$ excitation that is not generated by the TDDFT response to the core hole using a GGA exchange-correlation functional.

For completeness, we also show the direct RIXS contribution [Fig. 11(b)] for this model spectral function. The direct RIXS profile is again dominated by fluorescence above threshold. Below threshold, a Raman-like peak is still present but too weak to be observed in the color map. The indirect and direct contributions are combined in Fig. 11(c).

\section{SUMMARY AND OUTLOOK}

We have developed new methodology to calculate RIXS spectra from first principles with full momentum dependence for moderately correlated metallic materials. Compared to incident energy-dependent RIXS measurements on $\mathrm{BaFe}_{2} \mathrm{As}_{2}$, the calculations captured the overall trend of a crossover from Raman-like behavior below the XAS threshold to fluorescencelike above the XAS threshold. These results show that in both regions the loss spectra are dominated by the direct RIXS response and that the direct-channel Raman-like behavior is a consequence of the core-hole lifetime broadening. Accounting for the correlated response of the system through convolution with an effective spectral function incorporated secondary excitations and succeeded at capturing the detailed features of individual loss profiles very well. By pairing experimental and computational analysis, we uncovered the persistence of a $d d$ excitonic feature, previously undetected in the $\mathrm{Fe}$ pnictides. This feature is likely of mixed direct and indirect nature and offers a new means for probing electronic correlations of iron-based superconductors with RIXS.

Calculations begin by solving the Bethe-Salpeter equations, which limit excited states to a two-particle basis that is often insufficient for a detailed description of metallic and strongly correlated materials. We overcame this limitation through a quasiboson representation of secondary excitations by convolving the BSE spectra with effective many-body spectral functions. The spectral functions are formally derived as well from the quasiboson model and numerically evaluated by combining rt-TDDFT and a cumulant representation of the Green's function. This convolution greatly improved the agreement between calculated and measured XAS, XES, and direct-channel RIXS profiles and allowed ab initio evaluation of the indirect RIXS response incorporating the full periodicity of the system.

In the present work, we resorted to the incoherent approximation of the $\mathrm{KH}$ equation, a reasonable simplification when the RIXS loss profile closely reflects the nonresonant emission. However, this simplification is not a necessary approximation of our method. The full $\mathrm{KH}$ expression may be recovered by developing a single, more complex effective spectral function for the entire scattering process [61]. Generation of such a spectral function could be accomplished with a more detailed rt-TDDFT calculation incorporating both the absorption and emission processes and would extend the present methods to cases that show a stronger resonance effect than seen in $\mathrm{BaFe}_{2} \mathrm{As}_{2}$.

We constructed the effective spectral function using rt-TDDFT with a GGA exchange-correlation functional, which failed to generate the $d d$ excitation observed experimentally. This failure may be due to neglecting the photoelectron contribution to secondary excitations. With additional effort, the role of the photoelectron can be included [77]. Improving the treatment of electronic correlations should also help in this respect. For the underlying electronic structure, it would be desirable to advance from LDA to a quasiparticle self-consistent $G W$ level of theory. We envisage that the spectral functions could also be calculated at a more rigorous level of theory such as extensions of dynamical mean field theory [34,99].

We see great promise in this ability to calculate the manybody RIXS response of correlated metals from first principles and with full momentum resolution. However, there are limits to the applicability of our model with respect to electronic correlations. The approximation of accounting for many-body effects by convolving the BSE spectrum with a spectral function relies on the concept of a quasiparticle, which entirely breaks down in a Mott insulator. The intermediate case of a system with a quasiparticle remnant and Hubbard sidebands should be feasible. Beyond ironbased superconductors, other systems approachable with our technique could include certain vanadates, such as $\mathrm{SrVO}_{3}$ and $\mathrm{CaVO}_{3}$, as well as metallic $4 d$ and $5 d$ oxides including $\mathrm{Sr}_{2} \mathrm{RuO}_{4}$. Overdoped cuprates may also be amenable [100] but would require the use of supercells, which is computationally costly. Despite being considered Mott insulators, we suspect that an LDA $+U$ starting electronic structure could make $\mathrm{Sr}_{2} \mathrm{IrO}_{4}$ and $\mathrm{Sr}_{3} \mathrm{Ir}_{2} \mathrm{O}_{7}$ accessible as well [101]. Generally, though, multiorbital Hund's metals are the primary class that our method targets with key advantages being parameter-free inclusion of all orbitals, treatment of metal and ligand x-ray edges on equal footing, and access to arbitrary momentum transfer.

While we have explicitly considered only charge excitations such as electron-hole pairs and plasmons in this work, the approach is generalizable to other types of 
excitations. Recently, several studies have managed to resolve magnon excitations in metallic samples [13, 102-104]. Generating a spectral function with magnon and related spin excitation features would require performing a spin-resolved TDDFT calculation with specific inclusion of the spin dynamics of the core hole.

In summary, our work sets the basis for more detailed studies of the energy- and momentum-dependent RIXS response in multiorbital correlated metals. The methods presented here could yield a more refined understanding of the fascinating and complex physics of Hund's metals and materials with entangled spin and orbital excitations.

\section{ACKNOWLEDGMENTS}

K. G. was supported by the U.S. Department of Energy, Office of Science, Basic Energy Sciences, USA as part of the Computational Materials Science Program through the Center for Computational Design of Functional Strongly Correlated Materials and Theoretical Spectroscopy. J. J. K. acknowledges the support of the Theory Institute for Materials and Energies Science (TIMES) at SLAC which is funded by the U.S. Department of Energy, Office of Basic Energy Sciences, Division of Materials Sciences and Engineering, under Contract No. DE-AC02-76SF00515. J. P. and T. Schmitt acknowledge financial support through the Dysenos AG by Kabelwerke Brugg AG Holding, Fachhochschule Nordwestschweiz, and the Paul Scherrer Institut. J. P. acknowledges financial support by the Swiss National Science Foundation Early Postdoc Mobility fellowship Projects No. P2FRP2_171824 and No. P400P2 180744. The synchrotron radiation experiments have been performed at the ADRESS beam line of the Swiss Light Source at the Paul Scherrer Institut. Part of this research has been funded by the Swiss National Science Foundation through the D-A-CH program (SNSF Research Grant No. 200021L 141325). Work in Japan was supported by Grants-in-Aid for Scientific Research (KAKENHI) (No. JP18H05227, No. JP19H00649) from Japan Society for the Promotion of Science (JSPS), by the "Quantum Liquid Crystals" Grant-in-Aid for Scientific Research on Innovative Areas (No. JP19H05824) from the Ministry of Education, Culture, Sports, Science and Technology (MEXT) of Japan, and by JST CREST (No. JPMJCR19T5). K. G. thanks Joe Woicik and Ignace Jarrige for valuable discussions.

K. G. and J.P. contributed equally to this work.

\section{APPENDIX A: QUASIBOSON MODEL FOR XAS}

In this Appendix, we detail how to implicitly account for secondary excitations within single- or two-particle calculations of XAS and XES while avoiding much of the complexity of a fully correlated many-electron wave function. The principle approximation is to factor the full many-electron wave function into a product state comprised of the core level, the photoelectron, and the $N-1$ electron valence system. We then apply the quasiboson model to describe the secondary excitations within the valence system caused by both the core hole and photoelectron. Variants of this model are applied to corelevel spectroscopy in metals by Nozières and de Dominicis [90], Langreth [105], and Hedin and co-workers [91-93, 106] among others, which, in turn, rely on related work, such as by Mahan [89]. We arrive at Eq. (1), which approximates the many-electron absorption spectrum as a convolution over the BSE absorption spectrum and an effective spectral function, and provide additional details about using the quasiboson model to construct the effective spectral function. This summary relies heavily on the work of Campbell et al. [93], Kas, Rehr, and Curtis [77], and several references therein.

We start with the formal many-electron expression for the x-ray absorption coefficient:

$\mu(\omega)=-\frac{1}{\pi} \operatorname{Im}\left\langle\Psi_{0}\left|\hat{\Delta}^{\dagger} \frac{1}{\omega+E_{0}-\hat{H}+i \Gamma} \hat{\Delta}\right| \Psi_{0}\right\rangle$.

$\hat{H}$ is the fully interacting many-electron Hamiltonian with ground-state wave function $\left|\Psi_{0}\right\rangle$ and energy $E_{0}$. The dipole operator is

$$
\hat{\Delta}=\sum_{k}\langle k|\hat{d}| \alpha\rangle \hat{c}_{k}^{\dagger} \hat{a}+\text { H.c. }
$$

with $\hat{c}_{k}^{\dagger}$ the creation operator of the photoelectron $k$ and $\hat{a}$ the annihilation operator for the core hole $\alpha$. We invoke the core-excited Hamiltonian described by Langreth [105]:

$$
\hat{H}=\hat{H}_{v}+\epsilon_{\alpha} \hat{a}^{\dagger} \hat{a}+V \hat{a} \hat{a}^{\dagger},
$$

where $V$ is the interaction potential between the core hole and the other electrons and $\hat{H}_{v}$ is the Hamiltonian for the valence electrons and photoelectron. Neglecting explicit core-valence exchange, we may factor the ground-state many-electron wave function as $\left|\Psi_{0}\right\rangle=\left|\Phi_{0}\right\rangle|\alpha\rangle$ with $\left|\Phi_{0}\right\rangle$ the ground state of $\hat{H}_{v}$ in the presence of a filled core shell and absence of a photoelectron so that $\hat{H}_{v}\left|\Phi_{0}\right\rangle=E_{0}^{0}\left|\Phi_{0}\right\rangle$ and $E_{0}=E_{0}^{0}+\epsilon_{\alpha}$. The absorption coefficient then becomes

$$
\begin{aligned}
\mu(\omega)= & -\frac{1}{\pi} \operatorname{Im} \sum_{k_{1}, k_{2}}\left\langle\alpha\left|\hat{d}^{\dagger}\right| k_{1}\right\rangle\left\langle k_{2}|\hat{d}| \alpha\right\rangle \\
& \times\left\langle\Phi_{0}\left|\hat{c}_{k_{1}} \frac{1}{\omega-\left(\hat{H}_{v}^{\prime}-E_{0}^{\prime}\right)+i \gamma} \hat{c}_{k_{2}}^{\dagger}\right| \Phi_{0}\right\rangle .
\end{aligned}
$$

Primed quantities designate the presence of the core hole.

To proceed, we assume the Hamiltonian $\hat{H}_{v}$ may be separated as $\hat{H}_{v}=\hat{H}_{v}^{0}+\hat{h}_{p}+\hat{V}_{p v}$, where $\hat{H}_{v}^{0}$ is the Hamiltonian for the valence electrons neglecting the photoelectron, $\hat{h}_{p}$ acts on the photoelectron, and $\hat{V}_{p v}$ 
is the interaction between the photoelectron and valence electrons. Consistent with this partitioning, we factor out the orbital space of the photoelectron so that $\hat{c}_{k}^{\dagger}\left|\Phi_{0}\right\rangle=$ $\left|\Phi_{0}\right\rangle|k\rangle$. This factoring brings the propagator of Eq. (A4) to the form of an effective single-particle Green's function:

$$
G^{\mathrm{eff}}(\omega)=\left\langle\Phi_{0}\left|\frac{1}{\omega-\left(\hat{H}_{v}^{0 \prime}-E_{0}^{\prime}\right)-\hat{h}_{p}-\hat{V}_{p v}+i \gamma}\right| \Phi_{0}\right\rangle .
$$

These assumptions, which are commonly used [92], are reasonable when the photoelectron is not in the immediate vicinity of the Fermi level, though numerical work indicates they are generally reliable $[77,93,94]$.

To describe the response of the correlated valence electron system to the creation of a core hole and photoelectron, we use the following quasiboson effective Hamiltonian that couples the three subsystems (core hole, photoelectron, and valence electrons):

$$
\hat{H}_{v}^{\prime}=\hat{H}_{v}^{0}+\hat{h}_{h p}+\hat{V}_{h v}+\hat{V}_{p v} .
$$

The individual terms are

$$
\begin{aligned}
\hat{H}_{v}^{0} & =\sum_{q} \omega_{q} \hat{b}_{q}^{\dagger} \hat{b}_{q}, \\
\hat{h}_{h p} & =\epsilon_{\alpha} \hat{a}^{\dagger} \hat{a}+\sum_{k>k_{F}} \epsilon_{k} \hat{c}_{k}^{\dagger} \hat{c}_{k}+\hat{V}_{h p}, \\
\hat{V}_{h v} & =-\sum_{q} V_{\alpha \alpha}^{q}\left(\hat{b}_{q}^{\dagger}+\hat{b}_{q}\right) \hat{a} \hat{a}^{\dagger}, \\
\hat{V}_{p v} & =\sum_{q} \sum_{k_{1} k_{2}}\left[V_{k_{1} k_{2}}^{q} \hat{b}_{q}^{\dagger}+\left(V_{k_{1} k_{2}}^{q}\right)^{*} \hat{b}_{q}\right] \hat{c}_{k_{1}}^{\dagger} \hat{c}_{k_{2}} .
\end{aligned}
$$

The hole, the photoelectron, and their interaction are accounted for by $\hat{h}_{h p}$ and treated by solving the BetheSalpeter equation. Excitations within the valence electron system are described by $\hat{H}_{v}^{0}$ and the interactions of the valence system with the hole and photoelectron are given by $\hat{V}_{h v}$ and $\hat{V}_{p v}$, respectively. These interactions are expressed in terms of fluctuation potentials $V^{q}$ of mode $q$ of the valence system, which are described as quasibosons that are created (annihilated) by the bosonic operator $\hat{b}_{q}^{\dagger}\left(\hat{b}_{q}\right)$. In the present work, we intend these quasibosons to represent electron-hole pairs and plasmons, though they could also include Stoner excitations, magnons, localized collective excitations, and phonons. We note that these quasibosons are generated by both the core hole (intrinsic effects) and the photoelectron (extrinsic effects) and that there will be interference between these two contributions [77].

The quasiboson effective Hamiltonian has been discussed and solved before [77,91-93], finding that to second order in the fluctuation potentials the single-particle effective Green's function can be expressed as

$$
\begin{aligned}
G^{\mathrm{eff}}(\omega)= & e^{-a}\left[g_{p}(\omega)+\sum_{q}\left(\frac{V_{\alpha}^{q}}{\omega_{q}}\right)^{2} g_{p}\left(\omega-\omega_{q}\right)\right. \\
& \left.-2 \sum_{q} \frac{V_{\alpha}^{q}}{\omega_{q}} g_{p}\left(\omega-\omega_{q}\right) V_{p}^{q} g_{p}(\omega)\right] .
\end{aligned}
$$

In the above, $a=\sum_{q}\left(V_{\alpha}^{q} / \omega_{q}\right)^{2}$ quantifies the number of intrinsic shakeup excitations induced by the core hole, $g_{p}(\omega)$ is the dressed Green's function for the photoelectron, and $V_{\alpha}^{q}\left(V_{p}^{q}\right)$ are the fluctuation potentials of the valence system induced by the core hole (photoelectron). Under the condition that $G^{\text {eff }}$ is reasonably diagonal in the electronhole basis, Campbell et al. [93] show that the many-body absorption coefficient [Eq. (A4)] may be reduced to a convolution of the single-particle (or two-particle) absorption coefficient and a spectral function derived from $G^{\text {eff }}$, as in Eq. (1). One can then evaluate this Green's function using the cumulant expansion in the time domain [Eqs. (10)-(12)] and construct the effective spectral function from the imaginary part of $G^{\text {eff }}$.

\section{APPENDIX B: RIXS AS A CONVOLUTION OF XAS AND XES}

Standard one-particle and particle-hole approaches to core-level spectroscopy are unable to produce indirect RIXS contributions, because their excited states are explicitly limited to single-excitation wave functions. In this Appendix, we use the quasiboson model to obtain the indirect RIXS signal, as well as a more complete direct RIXS signal that includes secondary excitations. Following closely the work of Kas et al. [61], we achieve this result by factoring the full $\mathrm{KH}$ equation for the RIXS cross section into a convolution of the absorption and emission spectra and applying the results of the previous Appendix to dress the standard BSE spectra with secondary quasiboson excitations. This reduction of the $\mathrm{KH}$ formula into a convolution of the XAS and XES is not essential. One could instead obtain a single effective spectral function for the entire RIXS process. However, this task is considerably more complex.

The Green's function representation of the KramersHeisenberg formula is

$$
\begin{aligned}
& \frac{d^{2} \sigma}{d \omega_{i} d \omega_{o}} \\
& =-\frac{1}{\pi} \frac{\omega_{o}}{\omega_{i}} \operatorname{Im}\left\langle\Psi_{0}\left|\hat{\Delta}_{1}^{\dagger} G^{\alpha \dagger}\left(\xi_{1}\right) \hat{\Delta}_{2} G^{v}\left(\xi_{2}\right) \hat{\Delta}_{2}^{\dagger} G^{\alpha}\left(\xi_{1}\right) \hat{\Delta}_{1}\right| \Psi_{0}\right\rangle,
\end{aligned}
$$

where the Green's functions are given a superscript $\alpha(v)$ to indicate the presence of a hole in the core (valence) level and their energy arguments are $\xi_{1}=\omega_{i}+E_{0}$ and $\xi_{2}=$ $\omega_{l}+E_{0}$ with $E_{0}$ the ground-state energy and $\omega_{l}=\omega_{i}-\omega_{o}$ 
the energy loss. Assigning 1 to the incident photon and 2 to the outgoing photon, the dipole operators are

$$
\begin{aligned}
& \hat{\Delta}_{1}=\sum_{k}\left\langle k\left|\hat{d}_{1}\right| \alpha\right\rangle \hat{c}_{k}^{\dagger} \hat{a}+\text { H.c. } \\
& \hat{\Delta}_{2}=\sum_{v}\left\langle\alpha\left|\hat{d}_{2}\right| v\right\rangle \hat{a}^{\dagger} \hat{c}_{v}+\text { H.c. }
\end{aligned}
$$

Following the previous Appendix and indicating the ground-state wave function of the valence system as $\left|\Phi_{0}\right\rangle$, we may express the RIXS cross section as

$$
\begin{aligned}
\frac{d^{2} \sigma}{d \omega_{i} d \omega_{o}}= & -\frac{1}{\pi} \frac{\omega_{o}}{\omega_{i}} \operatorname{Im}\left[\sum_{k_{1} k_{2}}^{\text {unocc occ }} \sum_{v_{1} v_{2}}^{\alpha v_{1}} M_{2}^{v_{1}}\left(M_{2}^{v_{2} \alpha} M_{1}^{\alpha k_{1}}\right)^{*}\right. \\
& \left.\times M_{1}^{k_{2} \alpha}\left\langle\Phi_{0}\left|\hat{c}_{k_{1}} \Xi_{v_{1} v_{2}}\left(\xi_{1}, \xi_{2}\right) \hat{c}_{k_{2}}^{\dagger}\right| \Phi_{0}\right\rangle\right] .
\end{aligned}
$$

Here, we introduce the dipole matrix elements $M_{j}^{p h}=$ $\left\langle p\left|\hat{d}_{j}\right| h\right\rangle$ and the scattering operator

$$
\Xi_{v_{1} v_{2}}\left(\xi_{1}, \xi_{2}\right)=G^{\alpha \dagger}\left(\xi_{1}\right) \hat{c}_{v_{1}}^{\dagger} \hat{a} G^{v}\left(\xi_{2}\right) \hat{a}^{\dagger} \hat{c}_{v_{2}} G^{\alpha}\left(\xi_{1}\right) .
$$

Following the assumptions of a factorizable photoelectron state discussed in Appendix $\mathrm{A}$ and treating the

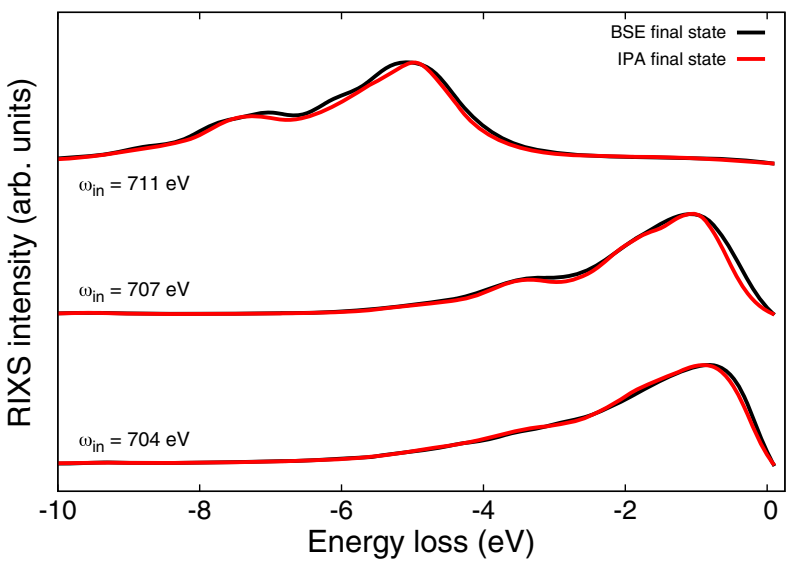

FIG. 12. RIXS spectra calculated using the full KramersHeisenberg formula by Eq. (8). Intermediate-state excitonic interactions are treated at the BSE level, while final-state excitonic interactions are evaluated either with the BSE kernel (black curves) or at the independent particle level (red curves). For all sets of curves, the intermediate (final) state HWHM broadening is $0.20 \mathrm{eV}(0.36 \mathrm{eV})$.

photoelectron as a spectator, the matrix elements related to the emission process may be factored out, yielding $[61,64,107]$

$$
\frac{d^{2} \sigma}{d \omega_{i} d \omega_{o}}=-\frac{1}{\pi} \frac{\omega_{o}}{\omega_{i}} \sum_{\alpha, v<k_{F}}\left|M_{2}^{\alpha v}\right|^{2} \operatorname{Im}\left[\sum_{k>k_{F}}\left|M_{1}^{k \alpha}\right|^{2}\left\langle\Phi_{0}\left|\hat{c}_{k} G^{\alpha^{\dagger}}\left(\xi_{1}\right) G^{v}\left(\xi_{2}\right) G^{\alpha}\left(\xi_{1}\right) \hat{c}_{k}^{\dagger}\right| \Phi_{0}\right\rangle\right] .
$$

With respect to insulating samples, metals offer two advantages that aid the above simplifications of the RIXS cross section. First, final-state excitonic interactions are weak, allowing us to neglect them, meaning that the excited electron essentially acts as a spectator during the scattering process. This approximation is very reasonable for many materials well above the absorption edge [64]. For metals, this approximation is quite good even in the proximity of a resonance, because the finalstate excitonic interactions are strongly screened. Figure 12 confirms the suitability of this approximation by comparing results of the Kramers-Heisenberg equation with and without final-state interactions. Second, while the RIXS intermediate state within insulating samples may couple only weakly to other excitations, such is not the case in metals, because secondary electron-hole pairs can have vanishing energy. Scattering with secondary excitations further attenuates the coherent contribution to the cross section.

To reduce the product of three Green's functions in Eq. (B5), we define effective Hamiltonians $H^{\alpha}$ and $H^{v}$ for the intermediate and final states, respectively, as

$$
\begin{aligned}
H^{\alpha} & =H^{0}+V_{\alpha}, \\
H^{v} & =H^{0}+V_{v}=H^{\alpha}+\Delta V_{v \alpha}, \\
\Delta V_{v \alpha} & =V_{v}-V_{\alpha} .
\end{aligned}
$$

With these definitions, the products of two Green's functions can be expressed as

$$
\begin{aligned}
G^{\alpha^{\dagger}} G^{v} & =\frac{\mathbb{T} G^{v}-G^{\alpha^{\dagger}}}{\Delta E^{*}}, \\
G^{v} G^{\alpha} & =\frac{G^{v} \mathbb{\mathbb { T }}^{\dagger}-G^{\alpha}}{\Delta E},
\end{aligned}
$$

where $\Delta E=\omega_{o}+\left(\epsilon_{\alpha}-\epsilon_{v}\right)+i \gamma$ is the energy difference between the arguments of the two Green's functions and $\mathbb{T}=\mathbb{1}+\Delta V_{v \alpha} G^{\alpha \dagger}$. With this expression, the product of three Green's functions becomes

$$
\begin{aligned}
& G^{\alpha \dagger} G^{v} G^{\alpha}=\frac{1}{2}\left[\left(G^{\alpha \dagger} G^{v}\right) G^{\alpha}+G^{\alpha \dagger}\left(G^{v} G^{\alpha}\right)\right] \\
& =\frac{\mathbb{\varpi}^{\dagger} G^{v} \mathbb{\prod}}{|\Delta E|^{2}}-\frac{1}{2}\left[\frac{\mathbb{\varpi}^{\dagger} G^{\alpha \dagger}}{|\Delta E|^{2}}+\frac{G^{\alpha} G^{\alpha \dagger}}{\Delta E}+\text { H.c. }\right] \text {. }
\end{aligned}
$$


Taking the imaginary part, we see that

$$
\operatorname{Im}\left[G^{\alpha \dagger} G^{v} G^{\alpha}\right]=\frac{\mathbb{T}^{\dagger} \operatorname{Im}\left[G^{v}\right] \mathbb{T}}{|\Delta E|^{2}},
$$

and Eq. (B5) reduces to

$$
\begin{aligned}
\frac{d^{2} \sigma}{d \omega_{i} d \omega_{o}}= & -\frac{1}{\pi} \frac{\left|\left\langle\alpha\left|d_{o}\right| v\right\rangle\right|^{2}}{\left|\omega_{o}+\epsilon_{\alpha}-\epsilon_{v}+i \Gamma\right|^{2}} \\
& \times \operatorname{Im}\left[\left\langle\alpha\left|D^{\dagger}\left(\omega_{i}\right) G^{v}\left(\omega_{l}+\epsilon_{v}\right) D\left(\omega_{i}\right)\right| \alpha\right\rangle\right],
\end{aligned}
$$

where we introduce the modified dipole transition operator $D(\omega)=\mathbb{T} \hat{d}$. Defining an effective absorptionlike coefficient

$\tilde{\mu}_{A}^{0}\left(\omega_{i} ; \omega_{l}\right)=-\frac{1}{\pi} \operatorname{Im}\left[\left\langle\alpha\left|D^{\dagger}\left(\omega_{i}\right) G^{v}\left(\omega_{l}+\epsilon_{v}\right) D\left(\omega_{i}\right)\right| \alpha\right\rangle\right]$,

the RIXS cross section simplifies to the convolution

$\frac{d^{2} \sigma}{d \omega_{i} d \omega_{o}}=\frac{\omega_{o}}{\omega_{i}} \int d \tilde{\omega} \frac{\mu_{E}^{0}(\tilde{\omega}) \tilde{\mu}_{A}^{0}\left(\omega_{i} ; \omega_{l}-\tilde{\omega}+\epsilon_{\alpha}\right)}{\left|\omega_{o}-\tilde{\omega}+i \Gamma\right|^{2}}$.

The difference between the standard absorption coefficient and the modified absorption coefficient is that the dipole operator $\hat{d}_{i}$ is replaced with the modified transition operator $D\left(\omega_{i}\right)=\left[1+\Delta V_{v \alpha} G^{\alpha \dagger}\left(\omega_{i}+\epsilon_{\alpha}\right)\right] \hat{d}_{i}$. When the potential difference $\Delta V_{v \alpha}$ between the intermediate and final states is weak and $\Delta V_{v \alpha} G^{\alpha \dagger}$ is small, the modified absorption coefficients can be approximated well by the standard absorption coefficients. This approximation brings the expression of the RIXS cross section to a convolution over absorption and emission coefficients:

$$
\frac{d^{2} \sigma}{d \omega_{i} d \omega_{o}}=\frac{\omega_{o}}{\omega_{i}} \int d \tilde{\omega} \frac{\mu_{E}^{0}(\tilde{\omega}) \mu_{A}^{0}\left(\omega_{l}-\tilde{\omega}+\epsilon_{\alpha}\right)}{\left|\omega_{o}-\tilde{\omega}+i \Gamma\right|^{2}} .
$$

We test the appropriateness of this result for $\mathrm{BaFe}_{2} \mathrm{As}_{2}$ in Fig. 13 by comparing the BSE evaluation of the full Kramers-Heisenberg formula [Eq. (B1)] to the approximation of the RIXS spectrum as a convolution over absorption and emission spectra [Eq. (B12)]. For this comparison, we use the standard BSE absorption and emission spectra $\mu_{A}^{0}$ and $\mu_{E}^{0}$, respectively, not including any additional secondary excitations. The agreement supports the use of our approximations leading to Eq. (B12). Finally, to account for the additional many-body effects causing the secondary excitations, we incorporate the physics of the quasiboson model by substituting the bare absorption and emission spectra with their dressed counterparts, each expressed as convolutions of the bare spectrum and an effective spectral function as in Eq. (1).

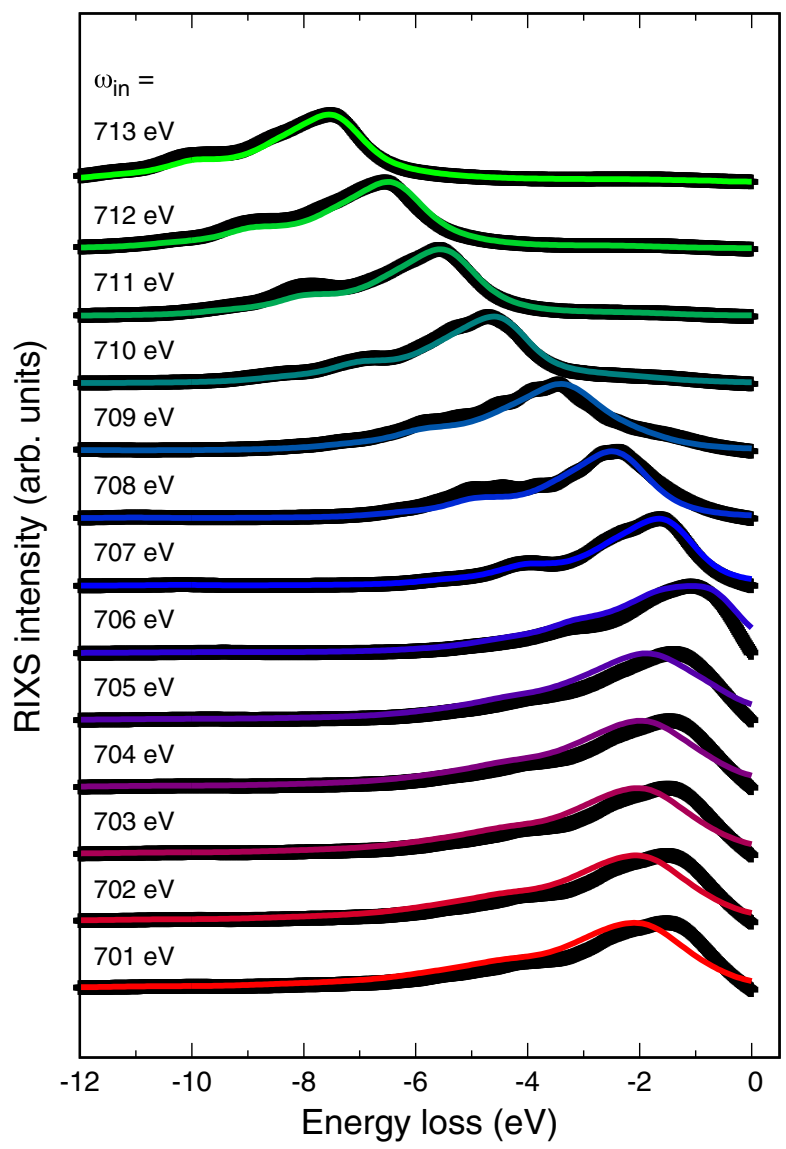

FIG. 13. Comparison of RIXS spectra calculated with the full Kramers-Heisenberg equation by Eq. (8) (black curves) and with the quasiboson model for the direct RIXS response in Eq. (13) using the bare BSE absorption and emission spectra $\mu_{a}^{0}$ and $\mu_{e}^{0}$, respectively (colored curves). Incident photon energies are indicated. All spectra are peak-height normalized to improve visual comparison. For all sets of curves, the intermediate (final) state HWHM broadening is $0.20 \mathrm{eV}(0.36 \mathrm{eV})$.

\section{APPENDIX C: LIFETIME EFFECT}

To further demonstrate that the Raman-like behavior of the RIXS spectra of $\mathrm{BaFe}_{2} \mathrm{As}_{2}$ below the XAS threshold originates from direct RIXS fluorescence processes that are enabled by the finite core-hole lifetime, Fig. 14 shows the calculated RIXS loss profiles below the XAS threshold for two values of the core-hole lifetime broadening. The below-threshold signal persists to much lower incident photon energy values for the larger core-level broadening. In fact, measuring the attenuation of the peak height while detuning below resonance could provide an experimental determination of the core-hole lifetime. In the simplified case of a single intermediate-state resonance, the denominator of the Kramers-Heisenberg equation shows that the signal intensity varies with the detuning $\Delta$ as $1 /\left[\Delta^{2}+(\gamma / 2)^{2}\right]$. However, the determination of the lifetime will be complicated by the presence of multiple resonances and effects that modify the Auger decay rate 


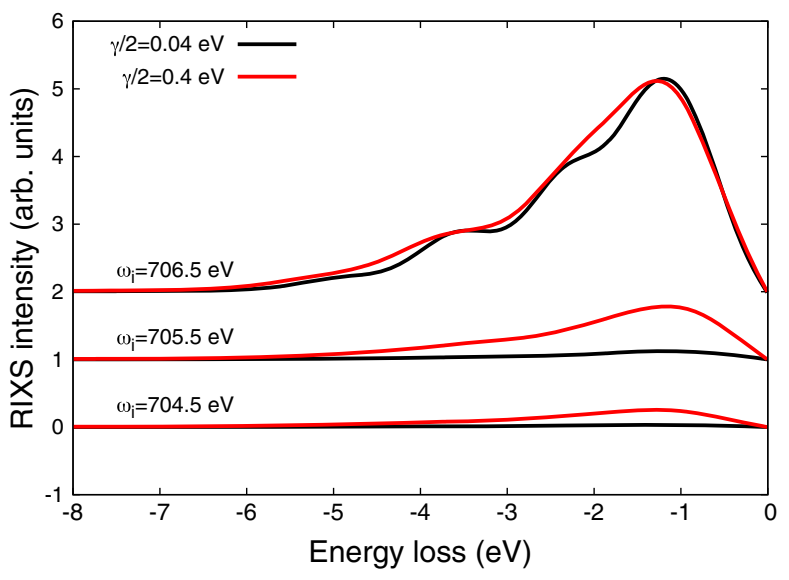

FIG. 14. Bethe-Salpeter equation calculations of the direct RIXS response of $\mathrm{BaFe}_{2} \mathrm{As}_{2}$ at and below the Fe $L_{3}$ XAS threshold for core-level broadenings of 0.04 and $0.4 \mathrm{eV}$. The longer core-hole lifetime (black curves) attenuates the RIXS signal below threshold more rapidly.

at resonance [82]. Nevertheless, such an analysis may be safely applicable for detunings sufficiently away from the resonance.

\section{APPENDIX D: COMPUTATIONAL DETAILS}

All BSE spectral calculations are performed with the OCEAN code [67] based on a DFT electronic structure obtained with Quantum ESPRESSO [69,70], which uses pseudopotentials and a plane-wave basis. The DFT calculations employed the LDA to the exchange-correlation functional and used norm-conserving pseudopotentials. For $\mathrm{Fe}$, we construct a pseudopotential with semicore states in valence.

For the x-ray absorption and emission calculations, a ground-state electronic charge density is obtained from a self-consistent-field (SCF) calculation with a $3 \times 3 \times 2$ $k$-point sampling. For the screening response, which we construct in a hybrid, real-space approach [108], a non-selfconsistent-field (NSCF) DFT calculation with $2 \times 2 \times 2$ $k$-point sampling and 240 unoccupied bands is sufficient. To evaluate the BSE spectrum, we construct a basis set of Bloch states by performing a NSCF DFT calculation with $7 \times 7 \times 5 k$-point sampling and 160 unoccupied bands for the absorption calculation. Both the SCF and NSCF calculations use plane-wave cutoff energies of $120 \mathrm{Ry}$ for the wave functions and 480 Ry for the density. Because of the need to treat the final-state valence excitonic states, the RIXS calculations require a much denser $k$-point sampling of $16 \times 16 \times 10$ with 160 unoccupied bands, as for the XAS calculation.

The final-state density of states (DOS) calculations are performed with the Quantum ESPRESSO code taking a $2 \times 2 \times 1$ supercell of the conventional unit cell. For these calculations, we use ultrasoft pseudopotentials and the GGA to the exchange-correlation functional. The $k$-point samplings are $2 \times 2 \times 3$ for the SCF calculation and $5 \times 5 \times 8$ for the NSCF calculation. The energy cutoffs are 40 Ry for the wave functions and 320 Ry for the charge density. For comparison to the XAS, the pseudopotential for one Fe site is replaced with an alternate pseudopotential containing a hole in the $2 p$ shell, while an extra electron is placed at the Fermi level to simulate the XAS final state. The ground-state DOS, for comparison to the XES, is calculated analogously, but without the use of a supercell or core-hole pseudopotential and extra electron.

The LDA-DFT-based DMFT calculations are performed using the Brookhaven National Lab Comscope suite. The electronic structure is initially solved at the all-electron DFT level using the FlapwMBPT code [109] using the LDA functional and a $6 \times 6 \times 4 k$-point mesh. A set of Wannier orbitals for states around the Fermi level are constructed with the Wannier90 [110] code using a frozen window of $\pm 20 \mathrm{eV}$ from the Fermi level and a disentanglement window of -20 to $60 \mathrm{eV}$ with respect to the Fermi level. The impurity problem is solved with the ComDMFT code [111] and a continuous-time quantum Monte Carlo algorithm with a simulation temperature of $300 \mathrm{~K}$. The ab initio determined Hubbard and Hund interactions are $U=$ $5.0 \mathrm{eV}$ and $J=0.8 \mathrm{eV}$, respectively, with a nominal $3 d^{6}$ occupancy for the double-counting correction [112].

The real-time TDDFT calculation, used to generate the valence charge density response to the sudden creation of an excitonic state, is performed with a modification [113] of the SIESTA DFT code [114]. A core hole is created at $t=0$ on a central iron atom of a $3 \times 3 \times 2$ supercell of $\mathrm{BaFe}_{2} \mathrm{As}_{2}$. The time evolution of the valence charge density response is performed with a Crank-Nicolson propagator using a time step of 0.3 a.u.

As we demonstrate in Sec. IV, the many-body spectral function associated with the sudden creation of a core-level exciton in $\mathrm{BaFe}_{2} \mathrm{As}_{2}$ can be approximated very well by a Doniach-Sunjic line shape, the expression for which is

$$
D S(\omega)=\frac{\cos \frac{\pi \alpha}{2}+(1-\alpha) \arctan \left(\frac{\omega-\omega_{0}}{\sigma}\right)}{\left[\sigma^{2}+\left(\omega-\omega_{0}\right)^{2}\right]^{(1-\alpha) / 2}} .
$$

The key parameter is the dimensionless quantity $\alpha$, which characterizes the asymmetry of the line shape. The other parameters are $\omega_{0}$, which indicates the peak position, and $\sigma$ that gives the symmetric contribution to the linewidth. Based on the spectral function obtained from the rt-TDDFT response, we use an asymmetry value of $\alpha=0.14$ and a linewidth of $\sigma=0.16 \mathrm{eV}$ for x-ray absorption. By convolving a DS line shape with the BSE-calculated XES and comparing to the experimental result, we obtain an asymmetry value of $\alpha=0.07$ for the XES process and the same linewidth of $\sigma=0.16 \mathrm{eV}$.

\section{APPENDIX E: EXPERIMENTAL METHODS}

Single crystals of $\mathrm{BaFe}_{2} \mathrm{As}_{2}$ are grown by stoichiometric melt as described in Ref. [115] and characterized with 
either resistivity or magnetization measurements. Fe- $L_{2,3}$ XAS and RIXS experiments are performed at the ADRESS beam line of the Swiss Light Source, Paul Scherrer Institut, Villigen PSI, Switzerland [19,20]. XAS is measured in both total fluorescence yield (TFY) and total electron yield (TEY) without observing significant differences. XAS and RIXS spectra are recorded at $15^{\circ}$ incidence angle relative to the sample surface. The RIXS spectrometer is set to a scattering angle of $130^{\circ}$. The total energy resolution is measured employing the elastic scattering of carbon-filled acrylic tape and is around $110 \mathrm{meV}$. The samples are mounted for XAS and RIXS experiments with the $a b$ plane perpendicular to the scattering plane and the $c$ axis lying in it and postcleaved in situ at a pressure better than $2.0 \times 10^{-10}$ mbar. We measure at $(0.31,0.31)$ using the orthorhombic unfolded crystallographic notation [116]. All the measurements are carried out at $14 \mathrm{~K}$ by cooling the manipulator with liquid helium.

$\mathrm{BaFe}_{2} \mathrm{As}_{2}$ has the tetragonal lattice structure (space group $I 4 / \mathrm{mmm}$ ) with $2 \mathrm{Fe}$ unit cell, and the relaxed parameters are $a=3.868 \AA$ and $c=12.378 \AA$.

[1] L.J. P. Ament, M. van Veenendaal, T. P. Devereaux, J. P. Hill, and J. van den Brink, Resonant Inelastic X-Ray Scattering Studies of Elementary Excitations, Rev. Mod. Phys. 83, 705 (2011).

[2] M. P. M. Dean, Insights into the High Temperature Superconducting Cuprates from Resonant Inelastic X-Ray Scattering, J. Magn. Magn. Mater. 376, 3 (2015).

[3] T. Schmitt, L.-C. Duda, M. Matsubara, M. Mattesini, M. Klemm, A. Augustsson, J.-H. Guo, T. Uozumi, S. Horn, R. Ahuja, A. Kotani, and J. Nordgren, Electronic Structure Studies of $\mathrm{V}_{6} \mathrm{O}_{13}$ by Soft X-Ray Emission Spectroscopy: Band-like and Excitonic Vanadium States, Phys. Rev. B 69, 125103 (2004).

[4] R. Fumagalli, L. Braicovich, M. Minola, Y. Y. Peng, K. Kummer, D. Betto, M. Rossi, E. Lefrancois, C. Morawe, M. Salluzzo, H. Suzuki, F. Yakhou, M. Le Tacon, B. Keimer, N. B. Brookes, M. M. Sala, and G. Ghiringhelli, Polarization-Resolved $\mathrm{Cu} \mathrm{l}_{3}$-Edge Resonant Inelastic $X$-Ray Scattering of Orbital and Spin Excitations in $\mathrm{NdBa}_{2} \mathrm{Cu}_{3} \mathrm{O}_{7-\delta}$, Phys. Rev. B 99, 134517 (2019).

[5] R.-P. Wang, B. Liu, R. J. Green, M. U. Delgado-Jaime, M. Ghiasi, T. Schmitt, M. M. van Schooneveld, and F. M.F. de Groot, Charge-Transfer Analysis of $2 p 3 d$ Resonant Inelastic X-ray Scattering of Cobalt Sulfide and Halides, J. Phys. Chem. C 121, 24919 (2017).

[6] H. Gretarsson, H. Suzuki, H. Kim, K. Ueda, M. Krautloher, B. J. Kim, H. Yavas, G. Khaliullin, and B. Keimer, Observation of Spin-Orbit Excitations and Hund's Multiplets in $\mathrm{Ca}_{2} \mathrm{RuO}_{4}$, Phys. Rev. B 100, 045123 (2019).

[7] B. W. Lebert, S. Kim, V. Bisogni, I. Jarrige, A. M. Barbour, and Y.-J. Kim, Resonant Inelastic X-Ray Scattering Study of $\mathrm{RuC}_{l 3}$ : A Progress Report, J. Phys. Condens. Matter 32, 144001 (2020).
[8] W. S. Lee, S. Johnston, B. Moritz, J. Lee, M. Yi, K. J. Zhou, T. Schmitt, L. Patthey, V. Strocov, K. Kudo, Y. Koike, J. van den Brink, T. P. Devereaux, and Z. X. Shen, Role of Lattice Coupling in Establishing Electronic and Magnetic Properties in Quasi-One-Dimensional Cuprates, Phys. Rev. Lett. 110, 265502 (2013).

[9] M. Rossi, R. Arpaia, R. Fumagalli, M. M. Sala, D. Betto, K. Kummer, G. M. De Luca, J. van den Brink, M. Salluzzo, N. B. Brookes, L. Braicovich, and G. Ghiringhelli, Experimental Determination of Momentum-Resolved Electron-Phonon Coupling, Phys. Rev. Lett. 123, 027001 (2019).

[10] L. Braicovich, L. J. P. Ament, V. Bisogni, F. Forte, C. Aruta, G. Balestrino, N. B. Brookes, G. M. De Luca, P. G. Medaglia, F. M. Granozio, M. Radovic, M. Salluzzo, J. van den Brink, and G. Ghiringhelli, Dispersion of Magnetic Excitations in the Cuprate $\mathrm{La}_{2} \mathrm{CuO}_{4}$ and $\mathrm{CaCuO}_{2}$ Compounds Measured Using Resonant X-Ray Scattering, Phys. Rev. Lett. 102, 167401 (2009).

[11] M. Le Tacon, G. Ghiringhelli, J. Chaloupka, M. M. Sala, V. Hinkov, M. W. Haverkort, M. Minola, M. Bakr, K. J. Zhou, S. Blanco-Canosa, C. Monney, Y. T. Song, G. L. Sun, C. T. Lin, G. M. De Luca, M. Salluzzo, G. Khaliullin, T. Schmitt, L. Braicovich, and B. Keimer, Intense Paramagnon Excitations in a Large Family of HighTemperature Superconductors, Nat. Phys. 7, 725 (2011).

[12] J. Kim, A. H. Said, D. Casa, M. H. Upton, T. Gog, M. Daghofer, G. Jackeli, J. van den Brink, G. Khaliullin, and B. J. Kim, Large Spin-Wave Energy Gap in the Bilayer Iridate $\mathrm{Sr}_{3} \mathrm{Ir}_{2} \mathrm{O}_{7}$ : Evidence for Enhanced Dipolar Interactions near the Mott Metal-Insulator Transition, Phys. Rev. Lett. 109, 157402 (2012).

[13] N. B. Brookes, D. Betto, K. Cao, Yi Lu, K. Kummer, and F. Giustino, Spin Waves in Metallic Iron and Nickel Measured by Soft X-Ray Resonant Inelastic Scattering, Phys. Rev. B 102, 064412 (2020).

[14] J. Schlappa, K. Wohlfeld, K. J. Zhou, M. Mourigal, M. W. Haverkort, V. N. Strocov, L. Hozoi, C. Monney, S. Nishimoto, S. Singh, A. Revcolevschi, J.-S. Caux, L. Patthey, H. M. Rnnow, J. van den Brink, and T. Schmitt, Spin-Orbital Separation in the Quasi-One-Dimensional Mott Insulator $\mathrm{Sr}_{2} \mathrm{CuO}_{3}$, Nature (London) 485, 82 (2012).

[15] C. Monney, K. J. Zhou, H. Cercellier, Z. Vydrova, M. G. Garnier, G. Monney, V. N. Strocov, H. Berger, H. Beck, T. Schmitt, and P. Aebi, Mapping of Electron-Hole Excitations in the Charge-Density-Wave System $1 t-\mathrm{TiSe}_{2}$ Using Resonant Inelastic X-Ray Scattering, Phys. Rev. Lett. 109, 047401 (2012).

[16] M. Hepting, L. Chaix, E. W. Huang, R. Fumagalli, Y. Y. Peng, B. Moritz, K. Kummer, N. B. Brookes, W. C. Lee, M. Hashimoto, T. Sarkar, J.-F. He, C. R. Rotundu, Y. S. Lee, R. L. Greene, L. Braicovich, G. Ghiringhelli, Z. X. Shen, T. P. Devereaux, and W. S. Lee, Three-Dimensional Collective Charge Excitations in Electron-Doped Copper Oxide Superconductors, Nature (London) 563, 374 (2018).

[17] M. P. M. Dean et al., Ultrafast Energy- and MomentumResolved Dynamics of Magnetic Correlations in the Photo-doped Mott Insulator $\mathrm{Sr}_{2} \mathrm{IrO}_{4}$, Nat. Mater. 15, 601 (2016). 
[18] Sergii Parchenko et al., Orbital Dynamics during an Ultrafast Insulator to Metal Transition, Phys. Rev. Research 2, 023110 (2020).

[19] G. Ghiringhelli, A. Piazzalunga, C. Dallera, G. Trezzi, L. Braicovich, T. Schmitt, V. N. Strocov, R. Betemps, L. Patthey, X. Wang, and M. Grioni, SAXES, a High Resolution Spectrometer for Resonant X-Ray Emission in the 400-1600 eV Energy Range, Rev. Sci. Instrum. 77, 113108 (2006).

[20] V. N. Strocov, T. Schmitt, U. Flechsig, T. Schmidt, A. Imhof, Q. Chen, J. Raabe, R. Betemps, D. Zimoch, J. Krempasky, X. Wang, M. Grioni, A. Piazzalunga, and L. Patthey, High-Resolution Soft X-Ray Beamline ADRESS at the Swiss Light Source for Resonant Inelastic X-Ray Scattering and Angle-Resolved Photoelectron Spectroscopies, J. Synchrotron Radiat. 17, 631 (2010).

[21] J. Dvorak, I. Jarrige, V. Bisogni, S. Coburn, and W. Leonhardt, Towards $10 \mathrm{meV}$ Resolution: The Design of an Ultrahigh Resolution Soft X-Ray RIXS Spectrometer, Rev. Sci. Instrum. 87, 115109 (2016).

[22] L. J. P. Ament, M. van Veenendaal, and J. van den Brink, Determining the Electron-Phonon Coupling Strength from Resonant Inelastic X-Ray Scattering at Transition Metal L-Edges, Europhys. Lett. 95, 27008 (2011).

[23] D. Benjamin, I. Klich, and E. Demler, Probing Competing and Intertwined Orders with Resonant Inelastic X-Ray Scattering in the Hole-Doped Cuprates, Phys. Rev. B 92, 035151 (2015).

[24] M. Kanasz-Nagy, Y. Shi, I. Klich, and E. A. Demler, Resonant Inelastic X-Ray Scattering as a Probe of Band Structure Effects in Cuprates, Phys. Rev. B 94, 165127 (2016).

[25] Yi Lu and M.W. Haverkort, Nonperturbative Series Expansion of Green's Functions: The Anatomy of Resonant Inelastic X-Ray Scattering in the Doped Hubbard Model, Phys. Rev. Lett. 119, 256401 (2017).

[26] A. Geondzhian and K. Gilmore, Demonstration of Resonant Inelastic X-Ray Scattering as a Probe of ExcitonPhonon Coupling, Phys. Rev. B 98, 214305 (2018).

[27] K. Tsutsui, T. Tohyama, and S. Maekawa, Mott Gap Excitations and Resonant Inelastic X-Ray Scattering in Doped Cuprates, Phys. Rev. Lett. 91, 117001 (2003).

[28] K. Okada and A. Kotani, Copper k and Oxygen k Resonant Inelastic X-Ray Scattering of One-Dimensional Cuprates, J. Phys. Soc. Jpn. 75, 044702 (2006).

[29] F. Vernay, B. Moritz, I. S. Elfimov, J. Geck, D. Hawthorn, T. P. Devereaux, and G. A. Sawatzky, Cu k-Edge Resonant Inelastic X-Ray Scattering in Edge-Sharing Cuprates, Phys. Rev. B 77, 104519 (2008).

[30] S. Kourtis, J. van den Brink, and M. Daghofer, Exact Diagonalization Results for Resonant Inelastic X-Ray Scattering Spectra of One-Dimensional Mott Insulators, Phys. Rev. B 85, 064423 (2012).

[31] C. Jia, K. Wohlfeld, Y. Wang, B. Moritz, and T. P. Devereaux, Using RIXS to Uncover Elementary Charge and Spin Excitations, Phys. Rev. X 6, 021020 (2016).

[32] A. Nocera, U. Kumar, N. Kaushal, G. Alvarez, E. Dagotto, and S. Johnston, Computing Resonant Inelastic X-Ray Scattering Spectra Using the Density Matrix Renormalization Group Method, Sci. Rep. 8, 11080 (2018).
[33] I. Josefsson, K. Kunnus, S. Schreck, A. Foehlisch, F. de Groot, P. Wernet, and M. Odelius, Ab Initio Calculations of X-Ray Spectra: Atomic Multiplet and Molecular Orbital Effects in a Multiconfigurational SCF Approach to the L-Edge Spectra of Transition Metal Complexes, J. Phys. Chem. Lett. 3, 3565 (2012).

[34] A. Hariki, M. Winder, T. Uozumi, and J. Kuneš, LDA + DMFT Approach to Resonant Inelastic X-Ray Scattering in Correlated Materials, Phys. Rev. B 101, 115130 (2020).

[35] D. Maganas, P. Kristiansen, L.-C. Duda, A. Knop-Gericke, S. DeBeer, R. Schloegl, and F. Neese, Combined Experimental and $A b$ Initio Multireference Configuration Interaction Study of the Resonant Inelastic X-Ray Scattering Spectrum of Co2, J. Phys. Chem. C 118, 20163 (2014).

[36] P. Zimmermann, R. J. Green, M. W. Haverkort, and F. M. F. de Groot, Quanty4RIXS: A Program for Crystal Field Multiplet Calculations of RIXS and RIXS-MCD Spectra Using Quanty, J. Synchrotron Radiat. 25, 899 (2018).

[37] Y. L. Wang, G. Fabbris, M. P. M. Dean, and G. Kotliar, Edrixs: An Open Source Toolkit for Simulating Spectra of Resonant Inelastic X-Ray Scattering, Comput. Phys. Commun. 243, 151 (2019).

[38] H. Y. Hwang, Y. Iwasa, M. Kawasaki, B. Keimer, N. Nagaosa, and Y. Tokura, Emergent Phenomena at Oxide Interfaces, Nat. Mater. 11, 103 (2012).

[39] S. Catalano, M. Gibert, J. Fowlie, J. Íñiguez, J.-M. Triscone, and J. Kreisel, Rare-Earth Nickelates $R \mathrm{NiO}$ 3: Thin Films and Heterostructures, Rep. Prog. Phys. 81, 046501 (2018).

[40] C. Pfleiderer, Superconducting Phases of $f$-Electron Compounds, Rev. Mod. Phys. 81, 1551 (2009).

[41] G. R. Stewart, Superconductivity in Iron Compounds, Rev. Mod. Phys. 83, 1589 (2011).

[42] Y. Maeno, H. Hashimoto, K. Yoshida, S. Nishizaki, T. Fujita, J. G. Bednorz, and F. Lichtenberg, Superconductivity in a Layered Perovskite without Copper, Nature (London) 372, 532 (1994).

[43] A. Bentien, S. Johnsen, G. K. H. Madsen, B. B. Iversen, and F. Steglich, Colossal Seebeck Coefficient in Strongly Correlated Semiconductor FeSb ${ }_{2}$, Europhys. Lett. 80, 17008 (2007).

[44] S. Shimizu, J. Shiogai, N. Takemori, S. Sakai, H. Ikeda, R. Arita, T. Nojima, A. Tsukazaki, and Y. Iwasa, Giant Thermoelectric Power Factor in Ultrathin FeSe Superconductor, Nat. Commun. 10, 825 (2019).

[45] Y. Song, Z. Yamani, C. Cao, Y. Li, C. Zhang, J. S. Chen, Q. Huang, H. Wu, J. Tao, Y. Zhu, W. Tian, S. Chi, H. Cao, Y.-B. Huang, M. Dantz, T. Schmitt, R. Yu, A. H. Nevidomskyy, E. Morosan, Q. Si, and P. Dai, A Mott Insulator Continuously Connected to Iron Pnictide Superconductors, Nat. Commun. 7, 13879 (2016).

[46] X. Deng, K. M. Stadler, K. Haule, A. Weichselbaum, J. von Delft, and G. Kotliar, Signatures of Mottness and Hundness in Archetypal Correlated Metals, Nat. Commun. 10, 2721 (2019).

[47] K. M. Stadler, G. Kotliar, A. Weichselbaum, and J. von Delft, Hundness versus Mottness in a Three-Band Hubbard-Hund Model: On the Origin of Strong Correlations in Hund Metals, Ann. Phys. (Amsterdam) 405, 365 (2019). 
[48] V. B. Zabolotnyy, D. S. Inosov, D. V. Evtushinsky, A. Koitzsch, A. A. Kordyuk, G. L. Sun, J. T. Park, D. Haug, V. Hinkov, A. V. Boris, C. T. Lin, M. Knupfer, A. N. Yaresko, B. Bchner, A. Varykhalov, R. Follath, and S. V. Borisenko, $(\pi, \pi)$ Electronic Order in Iron Arsenide Superconductors, Nature (London) 457, 569 (2009).

[49] C. E. Matt, N. Xu, B. Lv, J. Ma, F. Bisti, J. Park, T. Shang, C. Cao, Y. Song, A. H. Nevidomskyy, P. Dai, L. Patthey, N. C. Plumb, M. Radovic, J. Mesot, and M. Shi, $\mathrm{NaFe}_{0.56} \mathrm{Cu}_{0.44} \mathrm{As}$, Phys. Rev. Lett. 117, 097001 (2016).

[50] A. Charnukha, Optical Conductivity of Iron-Based Superconductors, J. Phys. Condens. Matter 26, 253203 (2014).

[51] M. Liu, L. W. Harriger, H. Luo, M. Wang, R. A. Ewings, T. Guidi, H. Park, K. Haule, G. Kotliar, S. M. Hayden, and P. Dai, Nature of Magnetic Excitations in Superconducting $\mathrm{BaFe}_{1.9} \mathrm{Ni}_{0.1} \mathrm{As}_{2}$, Nat. Phys. 8, 376 (2012).

[52] J. Laverock, B. Chen, K. E. Smith, R. P. Singh, G. Balakrishnan, M. Gu, J. W. Lu, S. A. Wolf, R. M. Qiao, W. Yang, and J. Adell, Resonant Soft-X-Ray Emission as a Bulk Probe of Correlated Electron Behavior in Metallic $\mathrm{Sr}_{x} \mathrm{Ca}_{1-x} \mathrm{VO}_{3}$, Phys. Rev. Lett. 111, 047402 (2013).

[53] D. E. McNally, X. Lu, J. Pelliciari, S. Beck, M. Dantz, M. Naamneh, T. Shang, M. Medarde, C. W. Schneider, V. N. Strocov, E. V. Pomjakushina, C. Ederer, M. Radovic, and T. Schmitt, Electronic Localization in $\mathrm{CaVo}_{3}$ Films via Bandwidth Control, npj Quantum Mater. 4, 6 (2019).

[54] K.-J. Zhou, Y.-B. Huang, C. Monney, X. Dai, V. N. Strocov, N.-L. Wang, Z.-G. Chen, C. Zhang, P. Dai, L. Patthey, J. van den Brink, H. Ding, and T. Schmitt, Persistent High-Energy Spin Excitations in Iron-Pnictide Superconductors, Nat. Commun. 4, 1470 (2013).

[55] J. Pelliciari, K. Ishii, M. Dantz, X. Lu, D. E. McNally, V. N. Strocov, L. Xing, X. Wang, C. Jin, H. S. Jeevan, P. Gegenwart, and T. Schmitt, Local and Collective Magnetism of $\mathrm{EuFe}_{2} \mathrm{As}_{2}$, Phys. Rev. B 95, 115152 (2017).

[56] J. Pelliciari, K. Ishii, Y. Huang, M. Dantz, X. Lu, P. OlaldeVelasco, V. N. Strocov, S. Kasahara, L. Xing, X. Wang, C. Jin, Y. Matsuda, T. Shibauchi, T. Das, and T. Schmitt, Reciprocity between Local Moments and Collective Magnetic Excitations in the Phase Diagram of $\mathrm{BaFe}_{2}\left(\mathrm{As}_{1 x} \mathrm{P}_{x}\right)_{2}$, Commun. Phys. 2, 139 (2019).

[57] F. A. Garcia, O. Ivashko, D. E. McNally, L. Das, M. M. Piva, C. Adriano, P. G. Pagliuso, J. Chang, T. Schmitt, and C. Monney, Anisotropic Magnetic Excitations and Incipient Néel Order in $\mathrm{Ba}\left(\mathrm{Fe}_{1-x} \mathrm{Mn}_{x}\right){ }_{2} \mathrm{As}_{2}$, Phys. Rev. B 99, 115118 (2019).

[58] J. Pelliciari, Y. Huang, T. Das, M. Dantz, V. Bisogni, P. O. Velasco, V. N. Strocov, L. Xing, X. Wang, C. Jin, and T. Schmitt, Intralayer Doping Effects on the High-Energy Magnetic Correlations in NaFeAs, Phys. Rev. B 93, 134515 (2016).

[59] J. Pelliciari, M. Dantz, Y. Huang, V. N. Strocov, L. Xing, X. Wang, C. Jin, and T. Schmitt, Presence of Magnetic Excitations in SmFeAsO, Appl. Phys. Lett. 109, 122601 (2016).

[60] M. C. Rahn, K. Kummer, N. B. Brookes, A. A. Haghighirad, K. Gilmore, and A. T. Boothroyd, Paramagnon Dispersion in $\beta$ - FeSe Observed by Fe L-Edge Resonant Inelastic XRay Scattering, Phys. Rev. B 99, 014505 (2019).
[61] J. J. Kas, J. J. Rehr, J. A. Soininen, and P. Glatzel, RealSpace Green's Function Approach to Resonant Inelastic X-Ray Scattering, Phys. Rev. B 83, 235114 (2011).

[62] C. J. Glover, T. Schmitt, M. Mattesini, M. Adell, L. Ilver, J. Kanski, L. Kjeldgaard, M. Agker, N. Mrtensson, R. Ahuja, J. Nordgren, and J.-E. Rubensson, Stationary and Dispersive Features in Resonant Inelastic Soft X-Ray Scattering at the Ge $3 p$ Resonances, J. Electron Spectrosc. Relat. Phenom. 173, 103 (2009).

[63] J. Vinson and J. J. Rehr, Ab Initio Bethe-Salpeter Calculations of the X-Ray Absorption Spectra of Transition Metals at the l-Shell Edges, Phys. Rev. B 86, 195135 (2012).

[64] J. Vinson, T. Jach, M. Müller, R. Unterumsberger, and B. Beckhoff, Quasiparticle Lifetime Broadening in Resonant $X$-Ray Scattering of $\mathrm{NH}_{4} \mathrm{NO}_{3}$, Phys. Rev. B 94, 035163 (2016).

[65] C. Vorwerk, F. Sottile, and C. Draxl, Excitation Pathways in Resonant Inelastic X-Ray Scattering of Solids, Phys. Rev. Research 2, 042003(R) (2020).

[66] A. Geondzhian, A. Sambri, G. M. De Luca, R. Di Capua, E. Di Gennaro, D. Betto, M. Rossi, Y. Y. Peng, R. Fumagalli, N. B. Brookes, L. Braicovich, K. Gilmore, G. Ghiringhelli, and M. Salluzzo, Large Polarons as Key Quasiparticles in $\mathrm{SrTiO}_{3}$ and $\mathrm{SrTiO}_{3}$-based heterostructures, Phys. Rev. Lett. 125, 126401 (2020).

[67] K. Gilmore, J. Vinson, E. L. Shirley, D. Prendergast, C. D. Pemmaraju, J. J. Kas, F. D. Vila, and J. J. Rehr, Efficient Implementation of Core-Excitation Bethe-Salpeter Equation Calculations, Comput. Phys. Commun. 197, 109 (2015).

[68] E. L. Shirley, Bethe-Salpeter Treatment of X-Ray Absorption including Core-Hole Multiplet Effects, J. Electron Spectrosc. Relat. Phenom. 144-147, 1187 (2005).

[69] P. Giannozzi et al., Quantum Espresso: A Modular and Open-Source Software Project for Quantum Simulations of Materials, J. Phys. Condens. Matter 21, 395502 (2009).

[70] P. Giannozzi et al., Advanced Capabilities for Materials Modelling with Quantum Espresso, J. Phys. Condens. Matter 29, 465901 (2017).

[71] E. L. Shirley, Ti 1s Pre-Edge Features in Rutile: A BetheSalpeter Calculation, J. Electron Spectrosc. Relat. Phenom. 136, 77 (2004).

[72] J. N. Hancock, R. Viennois, D. van der Marel, H. M. Rnnow, M. Guarise, P.-H. Lin, M. Grioni, M. M. Sala, G. Ghiringhelli, V. N. Strocov, J. Schlappa, and T. Schmitt, Evidence for Core-Hole-Mediated Inelastic X-Ray Scattering from Metallic $\mathrm{Fe}_{1.087}$ Te, Phys. Rev. B 82, 020513(R) (2010).

[73] S. L. Skornyakov, A. V. Efremov, N. A. Skorikov, M. A. Korotin, Yu. A. Izyumov, V. I. Anisimov, A. V. Kozhevnikov, and D. Vollhardt, Classification of the Electronic Correlation Strength in the Iron Pnictides: The Case of the Parent Compound $\mathrm{BaFe}_{2} \mathrm{As}_{2}$, Phys. Rev. B 80, 092501 (2009).

[74] G. Derondeau, F. Bisti, M. Kobayashi, J. Braun, H. Ebert, V. A. Rogalev, M. Shi, T. Schmitt, J. Ma, H. Ding, V. N. Strocov, and J. Minr, Fermi Surface and Effective Masses in Photoemission Response of the $\left(\mathrm{Ba}_{1 x} \mathrm{~K}_{x}\right) \mathrm{Fe}_{2} \mathrm{As}_{2}$ Superconductor, Sci. Rep. 7, 8787 (2017). 
[75] Y. Liang, J. Vinson, S. Pemmaraju, W. S. Drisdell, E. L. Shirley, and D. Prendergast, Accurate X-Ray Spectral Predictions: An Advanced Self-Consistent-Field Approach Inspired by Many-Body Perturbation Theory, Phys. Rev. Lett. 118, 096402 (2017).

[76] Y. Liang and D. Prendergast, Taming Convergence in the Determinant Approach for X-Ray Excitation Spectra, Phys. Rev. B 100, 075121 (2019).

[77] J. J. Kas, J. J. Rehr, and J. B. Curtis, Particle-Hole Cumulant Approach for Inelastic Losses in X-Ray Spectra, Phys. Rev. B 94, 035156 (2016).

[78] J. C. Woicik, C. Weiland, A. K. Rumaiz, M. T. Brumbach, J. M. Ablett, E. L. Shirley, J. J. Kas, and J. J. Rehr, Core Hole Processes in X-Ray Absorption and Photoemission by Resonant Auger-Electron Spectroscopy and FirstPrinciples Theory, Phys. Rev. B 101, 245105 (2020).

[79] J. C. Woicik, C. Weiland, C. Jaye, D. A. Fischer, A. K. Rumaiz, E. L. Shirley, J. J. Kas, and J. J. Rehr, ChargeTransfer Satellites and Chemical Bonding in Photoemission and X-Ray Absorption of $\mathrm{SrTio}_{3}$ and Rutile $\mathrm{Tio}_{2}$ : Experiment and First-Principles Theory with General Application to Spectroscopic Analysis, Phys. Rev. B 101, 245119 (2020).

[80] S. de Jong, Y. Huang, R. Huisman, F. Massee, S. Thirupathaiah, M. Gorgoi, F. Schaefers, R. Follath, J. B. Goedkoop, and M.S. Golden, High-Resolution, Hard X-Ray Photoemission Investigation of $\mathrm{BaFe}_{2} \mathrm{As}_{2}$ : Moderate Influence of the Surface and Evidence for a Low Degree of Fe3dAs4p Hybridization of Electronic States near the Fermi Energy, Phys. Rev. B 79, 115125 (2009).

[81] E. L. Shirley, Ab Initio Inclusion of Electron-Hole Attraction: Application to X-Ray Absorption and Resonant Inelastic X-Ray Scattering, Phys. Rev. Lett. 80, 794 (1998).

[82] M. Weinelt, A. Nilsson, M. Magnuson, T. Wiell, N. Wassdahl, O. Karis, A. Föhlisch, N. Mårtensson, J. Stöhr, and M. Samant, Resonant Photoemission at the $2 p$ Edges of Ni: Resonant Raman and Interference Effects, Phys. Rev. Lett. 78, 967 (1997).

[83] M. Magnuson, N. Wassdahl, A. Nilsson, A. Föhlisch, J. Nordgren, and N. Mårtensson, Resonant Auger Spectroscopy at the $L_{2,3}$ Shake-up Thresholds as a Probe of Electron Correlation Effects in Nickel, Phys. Rev. B 58, 3677 (1998).

[84] M. Finazzi, N. B. Brookes, and F. M. F. de Groot, $2 p 3 s 3 p$, $2 p 3 p 3 p$, and $2 p 3 s 3 s$ Resonant Auger Spectroscopy from NiO, Phys. Rev. B 59, 9933 (1999).

[85] J.-E. Rubensson, RIXS Dynamics for Beginners, J. Electron Spectrosc. Relat. Phenom. 110-111, 135 (2000).

[86] F. Gel'mukhanov and H. gren, Resonant X-Ray Raman Scattering, Phys. Rep. 312, 87 (1999).

[87] G. Ghiringhelli, N. B. Brookes, C. Dallera, A. Tagliaferri, and L. Braicovich, Sensitivity to Hole Doping of $\mathrm{Cu} \mathrm{L}_{3}$ Resonant Spectroscopies: Inelastic X-Ray Scattering and Photoemission of $\mathrm{La}_{2-x} \mathrm{Sr}_{x} \mathrm{CuO}_{4}$, Phys. Rev. B 76, 085116 (2007).

[88] P. W. Anderson, Infrared Catastrophe in Fermi Gases with Local Scattering Potentials, Phys. Rev. Lett. 18, 1049 (1967).
[89] G. D. Mahan, Excitons in Metals: Infinite Hole Mass, Phys. Rev. 163, 612 (1967).

[90] P. Nozières and C. T. de Dominicis, Singularities in the $X$-Ray Absorption and Emission of Metals. III. One-Body Theory Exact Solution, Phys. Rev. 178, 1097 (1969).

[91] W. Bardyszewski and L. Hedin, A New Approach to the Theory of Photoemission from Solids, Phys. Scr. 32, 439 (1985).

[92] L. Hedin, On Correlation Effects in Electron Spectroscopies and the GW Approximation, J. Phys. Condens. Matter 11, R489 (1999).

[93] L. Campbell, L. Hedin, J. J. Rehr, and W. Bardyszewski, Interference between Extrinsic and Intrinsic Losses in X-Ray Absorption Fine Structure, Phys. Rev. B 65, 064107 (2002).

[94] J. J. Kas, F. D. Vila, J. J. Rehr, and S. A. Chambers, RealTime Cumulant Approach for Charge-Transfer Satellites in X-Ray Photoemission Spectra, Phys. Rev. B 91, 121112 (R) (2015).

[95] J. C. Woicik, C. Weiland, C. Jaye, D. A. Fischer, A. K. Rumaiz, E. L. Shirley, J. J. Kas, and J. J. Rehr, ChargeTransfer Satellites and Chemical Bonding in Photoemission and X-Ray Absorption of $\mathrm{SrTio}_{3}$ and Rutile $\mathrm{Tio}_{2}$ : Experiment and First-Principles Theory with General Application to Spectroscopic Analysis, Phys. Rev. B 101, 245119 (2020).

[96] P. Cudazzo and L. Reining, Correlation Satellites in Optical and Loss Spectra, Phys. Rev. Research 2, 012032(R) (2020).

[97] K.-J. Zhou, M. Radovic, J. Schlappa, V. Strocov, R. Frison, J. Mesot, L. Patthey, and T. Schmitt, Localized and Delocalized Ti $3 d$ Carriers in $\mathrm{LaAlO}_{3} / \mathrm{SrTiO}_{3}$ Superlattices Revealed by Resonant Inelastic X-Ray Scattering, Phys. Rev. B 83, 201402(R) (2011).

[98] T. Nomura, Y. Harada, H. Niwa, K. Ishii, M. Ishikado, S. Shamoto, and I. Jarrige, Resonant Inelastic X-Ray Scattering Study of Entangled Spin-Orbital Excitations in Superconducting $\mathrm{PrFeAsO}_{0.7}$, Phys. Rev. B 94, 035134 (2016).

[99] J. T. Park, G. Friemel, Y. Li, J.-H. Kim, V. Tsurkan, J. Deisenhofer, H.-A. K. von Nidda, A. Loidl, A. Ivanov, B. Keimer, and D. S. Inosov, Magnetic Resonant Mode in the Low-Energy Spin-Excitation Spectrum of Superconducting $\mathrm{Rb}_{2} \mathrm{Fe}_{4} \mathrm{Se}_{5}$ Single Crystals, Phys. Rev. Lett. 107, 177005 (2011).

[100] K. P. Kramer et al., Band Structure of Overdoped Cuprate Superconductors: Density Functional Theory Matching Experiments, Phys. Rev. B 99, 224509 (2019).

[101] P. Liu and C. Franchini, Advanced First-Principle Modeling of Relativistic Ruddlesden-Popper Strontium Iridates, Appl. Sci. 11, 2527 (2021).

[102] H. Gretarsson, N. H. Sung, J. Porras, J. Bertinshaw, C. Dietl, J. A. N. Bruin, A. F. Bangura, Y. K. Kim, R. Dinnebier, Jungho Kim, A. Al-Zein, M. M. Sala, M. Krisch, M. Le Tacon, B. Keimer, and B. J. Kim, Persistent Paramagnons Deep in the Metallic Phase of $\mathrm{Sr}_{2-x} \mathrm{La}_{x} \mathrm{IrO}_{4}$, Phys. Rev. Lett. 117, 107001 (2016).

[103] M. Minola et al., Crossover from Collective to Incoherent Spin Excitations in Superconducting Cuprates Probed by Detuned Resonant Inelastic X-Ray Scattering, Phys. Rev. Lett. 119, 097001 (2017). 
[104] J. Pelliciari, S. Lee, K. Gilmore, J. Li, Y. Gu, A. Barbour, I. Jarrige, C. H. Ahn, F. J. Walker, and V. Bisogni, Tuning Spin Excitations in Magnetic Films by Confinement, Nat. Mater. 20, 188 (2021).

[105] D. C. Langreth, Singularities in the X-Ray Spectra of Metals, Phys. Rev. B 1, 471 (1970).

[106] L. Hedin, B. I. Lundqvist, and S. Lundqvist, New Structure in the Single-Particle Spectrum of an Electron Gas, Solid State Commun. 5, 237 (1967).

[107] J. Jiménez-Mier, J. van Ek, D. L. Ederer, T. A. Callcott, J. J. Jia, J. Carlisle, L. Terminello, A. Asfaw, and R. C. Perera, Dynamical Behavior of X-Ray Absorption and Scattering at the L Edge of Titanium Compounds: Experiment and Theory, Phys. Rev. B 59, 2649 (1999).

[108] E. L. Shirley, Local Screening of a Core Hole: A RealSpace Approach Applied to Hafnium Oxide, Ultramicroscopy 106, 986 (2006).

[109] A. L. Kutepov, V. S. Oudovenko, and G. Kotliar, Linearized Self-Consistent Quasiparticle GW Method: Application to Semiconductors and Simple Metals, Comput. Phys. Commun. 219, 407 (2017).

[110] A. A. Mostofi, J. R. Yates, G. Pizzi, Y.-S. Lee, I. Souza, D. Vanderbilt, and N. Marzari, An Updated Version of Wannier90: A Tool for Obtaining Maximally-Localised Wannier Functions, Comput. Phys. Commun. 185, 2309 (2014).

[111] S. Choi, P. Semon, B. Kang, A. Kutepov, and G. Kotliar, Comdmft: A Massively Parallel Computer Package for the
Electronic Structure of Correlated Electron Systems, Comput. Phys. Commun. 244, 277 (2019).

[112] Z. P. Yin, K. Haule, and G. Kotliar, Kinetic Frustration and the Nature of the Magnetic and Paramagnetic States in Iron Pnictides and Iron Chalcogenides, Nat. Mater. 10, 932 (2011).

[113] Y. Takimoto, F. D. Vila, and J. J. Rehr, Real-Time Time Dependent Density Functional Theory Approach for Frequency-Dependent Nonlinear Optical Response in Photonic Molecules, J. Chem. Phys. 127, 154114 (2007).

[114] J. M. Soler, E. Artacho, J. D. Gale, A. Garcia, J. Junquera, P. Ordejon, and D. Sanchez-Portal, The SIESTA Method for Ab Initio Order-n Materials Simulation, J. Phys. Condens. Matter 14, 2745 (2002).

[115] S. Kasahara, T. Shibauchi, K. Hashimoto, K. Ikada, S. Tonegawa, R. Okazaki, H. Shishido, H. Ikeda, H. Takeya, K. Hirata, T. Terashima, and Y. Matsuda, Evolution from Non-Fermi- to Fermi-Liquid Transport via Isovalent Doping in $\mathrm{BaFe}_{2}\left(\mathrm{As}_{1-x} \mathrm{P}_{x}\right)_{2}$ Superconductors, Phys. Rev. B 81, 184519 (2010).

[116] J. T. Park, D. S. Inosov, A. Yaresko, S. Graser, D. L. Sun, Ph. Bourges, Y. Sidis, Y. Li, J.-H. Kim, D. Haug, A. Ivanov, K. Hradil, A. Schneidewind, P. Link, E. Faulhaber, I. Glavatskyy, C. T. Lin, B. Keimer, and V. Hinkov, Symmetry of Spin Excitation Spectra in the Tetragonal Paramagnetic and Superconducting Phases of 122Ferropnictides, Phys. Rev. B 82, 134503 (2010). 